\title{
Cost Effectiveness of Treatments for Wet Age-Related Macular Degeneration
}

\author{
Paul Mitchell, ${ }^{1}$ Lieven Annemans, ${ }^{2}$ Richard White, ${ }^{3}$ Meghan Gallagher ${ }^{4}$ and Simu Thomas ${ }^{5}$ \\ 1 Discipline of Ophthalmology, University of Sydney, Westmead Hospital, Westmead, \\ New South Wales, Australia \\ 2 Department of Public Health, Faculty of Medicine, Ghent University, Ghent, Belgium \\ 3 Research Evaluation Unit, Oxford PharmaGenesis Ltd, Oxford, UK \\ 4 Novartis Pharma AG, Basel, Switzerland \\ 5 Novartis Pharmaceuticals Corporation, East Hanover, New Jersey, USA
}

\section{Contents}

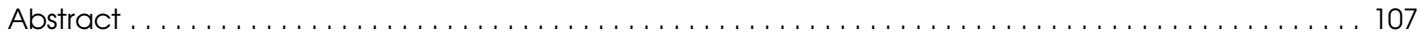

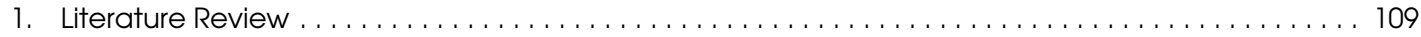

1.1 Methods ............................................. 109

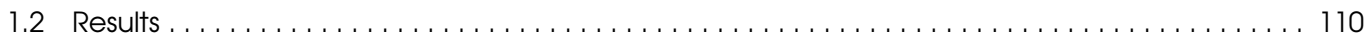

2. Comparison of Treatments with Best Supportive Care, Usual Care or Placebo. . . . . . . . . . . . . . 111

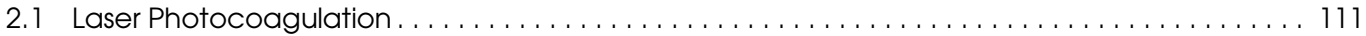

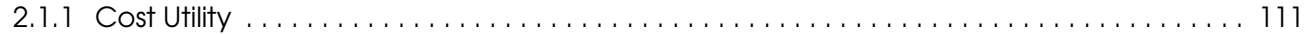

2.1 .2 Other Cost-Effectiveness Measures $\ldots \ldots \ldots \ldots \ldots \ldots \ldots \ldots \ldots \ldots \ldots \ldots \ldots \ldots \ldots \ldots \ldots \ldots$

2.2 Photodynamic Therapy with Verteporfin $\ldots \ldots \ldots \ldots \ldots \ldots \ldots \ldots \ldots \ldots \ldots \ldots$

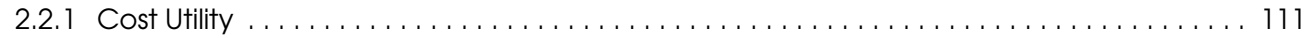

2.2 .2 Other Cost-Effectiveness Measures . . . . . . . . . . . . . . . . . . . . . . . . . . . 120

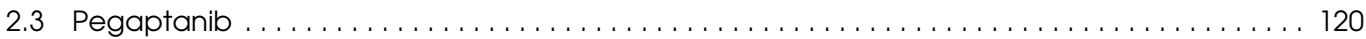

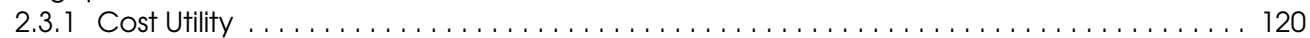

2.3.2 Other Cost-Effectiveness Measures . . . . . . . . . . . . . . . . . . . . . . . . . . . . . . . 120

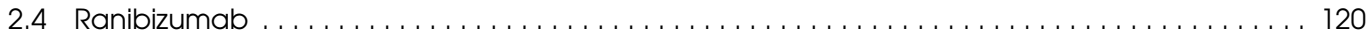

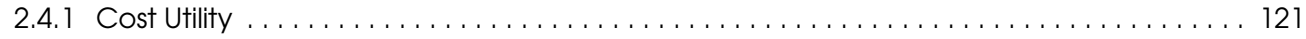

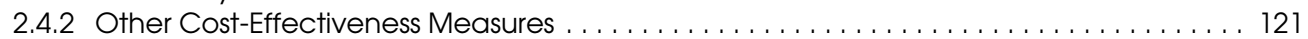

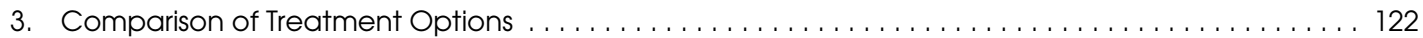

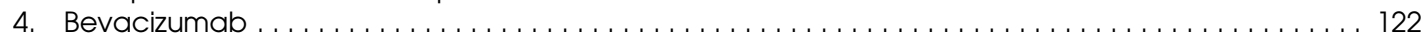

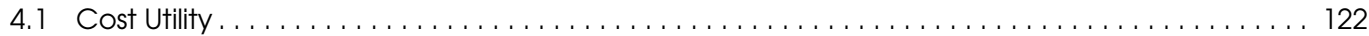

4.2 Other Cost-Effectiveness Measures. . . . . . . . . . . . . . . . . . . . . . . . . . . 123

4.3 Important Considerations for Intraocular Bevacizumab Use . . . . . . . . . . . . . . . . . . . 123

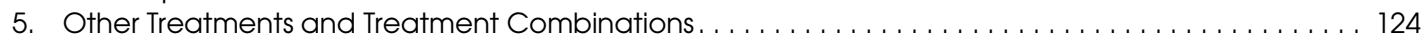

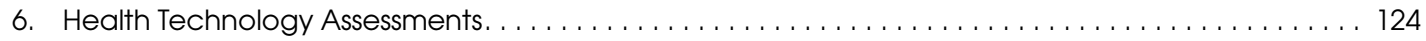

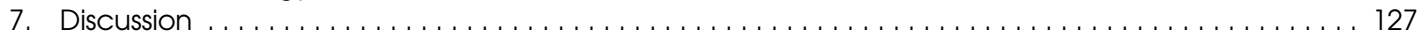

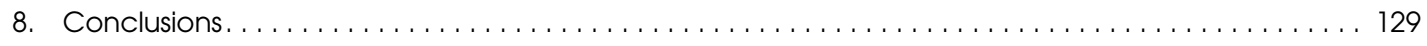

Age-related macular degeneration (AMD) is a leading cause of blindness in people aged $\geq 50$ years. Wet AMD in particular has a major impact on patient quality of life and imposes substantial burdens on healthcare systems. This systematic review examined the cost-effectiveness data for current 
therapeutic options for wet AMD. PubMed and EMBASE databases were searched for all articles reporting original cost-effectiveness analyses of wet AMD treatments. The Centre for Reviews and Dissemination and Cochrane Library databases were searched for all wet AMD health technology assessments (HTAs). Overall, 44 publications were evaluated in full and included in this review.

A broad range of cost-effectiveness analyses were identified for the most commonly used therapies for wet AMD (pegaptanib, ranibizumab and photodynamic therapy [PDT] with verteporfin). Three studies evaluated the cost effectiveness of bevacizumab in wet AMD. A small number of analyses of other treatments, such as laser photocoagulation and antioxidant vitamins, were also found.

Ranibizumab was consistently shown to be cost effective for wet AMD in comparison with all the approved wet AMD therapies (four of the five studies identified showed ranibizumab was cost effective vs usual care, PDT or pegaptanib); however, there was considerable variation in the methodology for cost-effectiveness modelling between studies. Findings from the HTAs supported those from the PubMed and EMBASE searches; of the seven HTAs that included ranibizumab, six (including HTAs for Australia, Canada and the UK) concluded that ranibizumab was cost effective for the treatment of wet AMD; most compared ranibizumab with PDT and/or pegaptanib. By contrast, HTAs at best generally recommended pegaptanib or PDT for restricted use in subsets of patients with wet AMD. In the literature analyses, pegaptanib was found to be cost effective versus usual/best supportive care (including PDT) or no treatment in one of five studies; the other four studies found pegaptanib was of borderline cost effectiveness depending on the stage of disease and time horizon. PDT was shown to be cost effective versus usual/best supportive care or no treatment in five of nine studies; two studies showed that PDT was of borderline cost effectiveness depending on baseline visual acuity, and two showed that PDT was not cost effective. We identified no robust studies that properly evaluated the cost effectiveness of bevacizumab in wet AMD.

Age-related macular degeneration (AMD)/agerelated maculopathy (ARM) refers to pathological changes in the central area of the retina that can occur in people aged $\geq 50$ years. ${ }^{[1]}$ Many people who have these changes do not experience symptoms; however, progressive alterations can lead to late-stage ARM (this stage is then referred to as AMD) and vision loss. The form of late-stage AMD most likely to cause blindness is neovascular exudative disease (also termed wet AMD). ${ }^{[2]}$ Wet AMD can be designated classic or occult according to its features on fluorescein angiography, with AMD lesions classified as either $100 \%$ classic, predominantly classic (in which choroidal neovascularization [CNV] accounts for at least $50 \%$ of the lesion), minimally classic (where CNV accounts for part of but $<50 \%$ of the lesion) or occult (where there is no CNV). ${ }^{[2]}$

Symptoms of wet AMD often begin with central visual blurring, distortion (metamorphopsia) or a dark central patch (scotoma), although, if only one eye is affected, these features may not be noticed for some time. When the second eye becomes affected, patients suddenly lose the ability to read, drive or see fine details such as facial expressions and features. AMD (both wet and dry) is one of the leading causes of blindness in the Western world and, because AMD affects older people, its prevalence is set to increase with the rising average age of populations. Indeed, it has been estimated that by 2020 the prevalence of AMD will be three times greater than it was in 
1995 , with up to 7.5 million people aged $>65$ years likely to be affected by AMD-related visual impairment. ${ }^{[3]}$

Costs associated with visual impairment are considerable, and include medical care, loss of income and paid home help. A 2006 study in France $^{[4]}$ estimated that total country-wide nonmedical costs of visual impairment were $€ 9800$ million per annum. A similar study in Australia ${ }^{[5]}$ estimated that the 2004 cost for vision disorders was Australian dollars (\$A)9850 million. A further study estimated total annual non-medical costs of visual impairment to be $€ 10749$ million, $€ 9214$ million, $€ 12069$ million and $€ 15180$ million in France, Germany, Italy and the UK, respectively (year 2004 values). ${ }^{[6]}$

The most commonly used current treatment options for wet AMD are laser photocoagulation, photodynamic therapy (PDT) and intravitreal injections with inhibitors of vascular endothelial growth factor (VEGF)-A. Laser photocoagulation aims to prevent further vision loss by destroying the neovascular complex; PDT is based on a similar concept but involves using a photosensitive agent (usually verteporfin), given intravenously. This agent is activated by laser light (689 $\mathrm{nm}$ wavelength) directed to the CNV lesion and causes damage to vascular endothelial cells and thrombotic occlusion of the blood vessels, while reducing concurrent damage to the overlying retina. New pharmaceutical treatments approved for wet AMD are directed against VEGF-A; for example, ranibizumab (a monoclonal antibody fragment) and pegaptanib (a synthetic oligonucleotide) inhibit the biological activity of VEGF-A, thereby aiming to reduce angiogenesis and stall AMD progression. ${ }^{\text {[7-9] }}$

Anti-VEGF treatments for wet AMD have recently been the subject of intense scrutiny; although they have revolutionized the treatment of the condition, offering increases in visual acuity over traditional therapies in the majority of patients, they come at increased costs. Several economic analyses have thus been conducted by both health economists and ophthalmologists to evaluate the cost effectiveness of wet AMD treatments; in addition, because of the implications for medical and social care, healthcare authorities around the world have commissioned their own health technology assessments (HTAs). The aim of this review is to examine the available costeffectiveness data for the current therapeutic options for wet AMD by conducting a systematic search of the scientific literature and reimbursement authority-authorized HTAs.

\section{Literature Review}

\section{1 Methods}

A search of the PubMed and EMBASE databases was conducted in November 2009 using the following search terms: (i) macular degeneration[MeSH] AND (cost-benefit analysis[MeSH] OR economics[MeSH]); (ii) [wet OR neovascular] AND ‘macular degeneration' AND cost. No other limits were imposed on the search. All articles published before or during November 2009 were eligible for screening. Titles and abstracts of all articles were screened by two reviewers.

In addition, the HTA databases and UK NHS Economic Evaluation Databases (EED) at the Centre for Reviews and Dissemination (CRD), University of York, and the Cochrane Library were searched in May 2010 to identify HTAs using the following search terms: (Macula OR Macular OR Retina OR Retinal OR Subretinal OR Choroidal OR AMD OR ARMD). All HTAs published before or during May 2010 were eligible for screening. The titles and/or abstracts of all identified records were screened manually by two reviewers for potential inclusion in the review.

The full texts of the identified publications were obtained and screened manually to select those that contained novel cost-effectiveness assessments, and those that were full HTAs (regardless of whether the HTAs contained original cost-effectiveness assessments). Data from publications that met these criteria were extracted into the tables included in this review. Studies that reported purely cost data (e.g. burden-ofillness studies, cost-of-illness studies) or that were reviews of previous cost-effectiveness evaluations, were excluded. Where possible, foreign language publications were translated in order to gather the required information. 


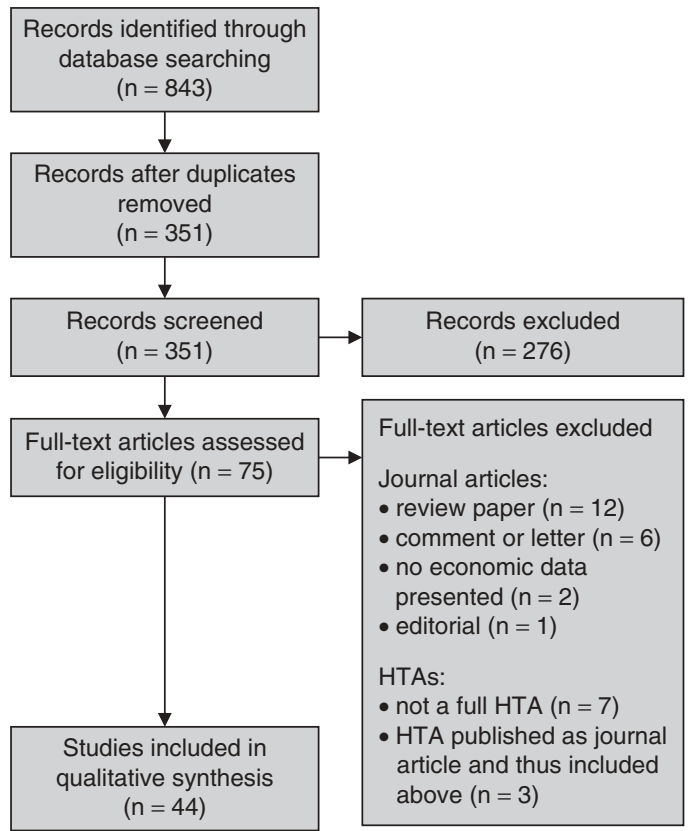

Fig. 1. Flow of the systematic review process. HTA=health technology assessment.

\subsection{Results}

The flow of the systematic review process is presented in figure 1. In total, 843 publications were identified by searching the PubMed, EMBASE, CRD and Cochrane Library databases. After eliminating duplicates, 351 unique publications were identified, and 75 warranted further investigation. Of the 276 articles excluded based on the title/abstract, most were not cost-effectiveness studies/HTAs of wet AMD treatments. The remainder were opinion, comment or letter articles, review articles, foreign language publications with no translation, or did not contain any pharmacoeconomic data.

The studies identified in our analysis employed a broad range of methodologies, perspectives and assumptions, which made comparisons between studies difficult. A summary of the studies is provided in the Supplemental Digital Content 1, http://links.adisonline.com/PCZ/A100. Most studies expressed the results in terms of cost per QALY gained (i.e. most studies involved costutility analyses). Other cost-effectiveness measures included the cost per vision-year gained, cost per line-year gained (using either the Snellen or, mostly, the ETDRS chart) [see table I for full study names] and cost per case of blindness prevented. The majority of economic analyses used a second (betterseeing) eye model. This model assumes treatment is not initiated until the second eye is affected; effective treatment thus has a greater impact on visual

Table I. Study acronyms and names

\begin{tabular}{|c|c|}
\hline $\begin{array}{l}\text { Study } \\
\text { acronym }\end{array}$ & Study name \\
\hline$\overline{\mathrm{ABC}}$ & Avastin (Bevacizumab) for Choroidal neovascular age-related macular degeneration \\
\hline ANCHOR & $\begin{array}{l}\text { ANti-VEGF antibody for the treatment of predominantly classic CHORoidal neovascularization in age-related macular } \\
\text { degeneration }\end{array}$ \\
\hline AREDS & Age-Related Eye Disease Study \\
\hline CATT & Comparison of Age-related macular degeneration Treatments Trial \\
\hline ETDRS & Early Treatment Diabetic Retinopathy Study \\
\hline IVAN & A randomised controlled trial of alternative treatments to Inhibit VEGF in Age-related choroidal Neovascularization \\
\hline MARINA & $\begin{array}{l}\text { Minimally classic/occult trial of the Anti-VEGF antibody Ranibizumab In the treatment of Neovascular Age-related macular } \\
\text { degeneration }\end{array}$ \\
\hline MICMAC & MICroeconomics of MACular degeneration \\
\hline MPS & Macular Photocoagulation Study \\
\hline PIER & $\begin{array}{l}\text { Phase 3b, multi-centre, randomized, double-masked, sham Injection-controlled study of the Efficacy and safety of } \\
\text { Ranibizumab in subjects with subfoveal choroidal neovascularization with or without classic CNV secondary to AMD }\end{array}$ \\
\hline PrONTO & Prospective Optical coherence tomography imaging of patients with Neovascular AMD Treated with intra-Ocular ranibizumab \\
\hline TAP & Treatment of Age-related macular degeneration with Photodynamic therapy \\
\hline VISION & VEGF Inhibition Study In Ocular Neovascularization \\
\hline
\end{tabular}

$\overline{\mathbf{A M D}}=$ age-related macular degeneration; $\mathbf{C N V}=$ choroidal neovascularization; VEGF = vascular endothelial growth factor. 
acuity than if the first eye to be affected were treated (when the unaffected eye compensates for loss of vision in the affected eye). Only two studies included a first-eye model, and in both cases this was evaluated alongside a second-eye model.

The major factors that had an impact on cost effectiveness were the timeframes used in the model, and the inclusion or exclusion of indirect costs of treatment (i.e. societal costs related to blindness, including caregiver costs). The timeframes taken into account by the models ranged from 1 year to a lifetime, and were generally extrapolated from 1-5 years of treatment. The types of wet AMD assessed most frequently were classic or predominantly classic AMD, but many studies included all types of the disease. Table II provides an overview of the methodology of the cost-utility studies included in the review. Treatment of the predominantly classic form of AMD was typically associated with lower incremental cost-effectiveness ratios (ICERs) than treatment of the occult disease form.

\section{Comparison of Treatments with Best Supportive Care, Usual Care or Placebo}

\section{1 Laser Photocoagulation}

\subsubsection{Cost Utility}

Laser photocoagulation was shown to be a cost-effective treatment option for wet AMD in the US in three studies based on data from the MPS group. Costs per QALY gained for laser photocoagulation compared with no treatment or with observation were \$US5629-23176 over time horizons of $11-14$ years (table III). ${ }^{[10-12]}$

\subsubsection{Other Cost-Effectiveness Measures}

Data from a number of clinical trials, along with allowable Medicare amounts for 2006, were used to determine cost effectiveness of laser photocoagulation in the US by costs per Snellen lineyear gained; the cost per Snellen line-year gained for extrafoveal disease was \$US77, and for juxtafoveal and subfoveal disease was \$US176. ${ }^{[32]}$

\subsection{Photodynamic Therapy with Verteporfin}

Before the availability of VEGF inhibitors for the treatment of wet AMD, PDT was the mainstay of therapy, and so its cost effectiveness has been evaluated extensively. Most analyses used efficacy data from the TAP study, a randomized double-masked trial of 609 patients with CNV, which showed that PDT with verteporfin could reduce the risk of vision loss ( $\geq 15$ letters) over 12 months compared with placebo. ${ }^{[33]}$

Most studies that modelled time horizons of $\geq 5$ years have shown PDT with verteporfin to be cost effective $(<£ 30000$ or \$US50 000-100 000 per QALY gained) compared with usual care, placebo or routine clinical practice in Canada, Switzerland, the UK and the US. Tables III and IV present summaries of the cost-effectiveness outcomes for PDT compared with usual care, no treatment or placebo.

\subsubsection{Cost Utility}

Using 5-year efficacy data, the estimated cost per QALY gained was $<£ 30000$ in the UK over a 10 -year time horizon ${ }^{[14]}$ and $<\$$ US50 000 in the US over a 12-year time horizon for predominantly classic/classic AMD. ${ }^{[1,15]}$ A Canadian HTA evaluated PDT from a societal perspective over an 8 -year timeframe, and also judged it to be cost effective $(<\$ C a n 50000)$ both for predominantly classic CNV and for classic and occult $\mathrm{CNV}^{\text {; }[13]}$ however, Sharma et al. ${ }^{[19]}$ evaluated PDT from a third-party payer's perspective using 1- to 2-year TAP data and found PDT to be of poor-tomodest cost effectiveness based on an 11-year timeframe.

PDT was found to be more likely to be cost effective when given early in the course of the disease to patients with better visual acuity; a study using a 7-year evaluation period in an Australian setting ${ }^{[17]}$ and a 5-year period in the $\mathrm{UK}^{[20]}$ both showed that PDT was borderline cost effective versus placebo for patients with 'reasonable' initial visual acuity $(6 / 12[20 / 40])^{1}$ at treatment

1 The standard definition of normal visual acuity (20/20 or 6/6 vision) is the ability to resolve a spatial pattern separated by a visual angle of 1 minute of arc. A person with a visual acuity of 6/12 (20/40) can resolve the same pattern at a distance of 6 metres (20 feet) as a person with 'normal' visual acuity can at 12 metres (40 feet). 
Table II. Summary of the methods of the reviewed cost-utility papers

\begin{tabular}{|c|c|c|c|c|}
\hline $\begin{array}{l}\text { Study, country, currency, } \\
\text { y of values }\end{array}$ & Perspective (comparator) & No. of $t x$ & $\begin{array}{l}\text { Annual disc. } \\
\text { rate }(\%)\end{array}$ & Model type \\
\hline \multicolumn{5}{|c|}{ Cost utility of laser photocoagulation vs UC, no tx or PL } \\
\hline Brown et al., ${ }^{[10]}$ US, \$US, 1999 & $\begin{array}{l}\text { Third-party payer; } 1 \text { y of tx; } \\
11-y \text { timeframe (no tx) }\end{array}$ & NR & 3.0 & 2nd eye \\
\hline Brown et al., ${ }^{[11]}$ US, \$US, 2005 & $\begin{array}{l}\text { Third-party payer - health insurance; } \\
1 \text { y of tx; } 12-y \text { timeframe (no tx) }\end{array}$ & 1.5 over $1 \mathrm{y}$ & 3.0 & 2nd eye \\
\hline Busbee et al.,[12] US, \$US, 2001 & $\begin{array}{l}\text { Third-party payer - health insurance; } \\
\text { up to } 5 \mathrm{y} \text { of tx; } 14-\mathrm{y} \text { timeframe } \\
\text { (observation) }\end{array}$ & 2 & 3.0 & NR \\
\hline \multicolumn{5}{|c|}{ Cost utility of PDT vs verteporfin with UC, no tx or PL } \\
\hline $\begin{array}{l}\text { Larouche and Rochon (AETMIS - } \\
\text { HTA), }{ }^{[13]} \text { Canada, \$Can, NR }\end{array}$ & Societal; 3 y of tx; 8-y timeframe (no tx) & $\begin{array}{l}3.4 \text { in y } 1 \\
2.1 \text { in y } 2 \\
1 \text { in y } 3\end{array}$ & 3.0 & Combined eye \\
\hline Bansback et al., ${ }^{[14]}$ UK, £, NR & $\begin{array}{l}\text { NR; up to } 10 y \text { of tx; 2-, 5- and 10-y } \\
\text { timeframes (BSC) }\end{array}$ & As per TAP & 3.5 & 2nd eye \\
\hline Brown et al.., ${ }^{[11]}$ US, \$US, 2005 & $\begin{array}{l}\text { Third-party payer - health insurance; } \\
12-y \text { timeframe (no tx) }\end{array}$ & 8.1 over $12 y$ & 3.0 & 2nd eye \\
\hline Brown et al., ${ }^{[15]}$ US, \$US, 2004 & $\begin{array}{l}\text { Third-party payer - health insurance; } \\
\text { 12-y timeframe }(P L)\end{array}$ & 8.1 over $12 y$ & 3.0 & 2nd eye \\
\hline Donati, ${ }^{[16]}$ Switzerland, $€$, NR & $\begin{array}{l}\text { Healthcare system; societal; 3y of tx; } \\
\text { 3-y timeframe }(P L)\end{array}$ & 3 over $3 y$ & 0.0 & NR \\
\hline $\begin{array}{l}\text { Hopley et al., }{ }^{[17]} \text { Australia, } \\
\$ A / £, 2003\end{array}$ & $\begin{array}{l}\text { Third-party payer; } 7 \mathrm{y} \text { of } \mathrm{tx} \\
\text { 7-y timeframe }(\mathrm{PL})\end{array}$ & 10.9 over $7 y$ & 6.0 & 2nd eye \\
\hline $\begin{array}{l}\text { Meads et al. (HTA), }{ }^{[18]} \text { UK, } \\
£, 2001\end{array}$ & $\begin{array}{l}\text { Health service and societal; } 2 \mathrm{y} \text { of } \mathrm{tx} \text {; } \\
2-\mathrm{y} \text { timeframe (BSC) }\end{array}$ & $1-8$ over $2 y$ & 0.0 & 2nd eye \\
\hline $\begin{array}{l}\text { Sharma et al., }{ }^{[19]} \text { Canada, } \\
\text { \$US, NR }\end{array}$ & $\begin{array}{l}\text { Third-party payer; } 2-\text { and } 11-y \\
\text { timeframes }(\mathrm{PL})\end{array}$ & 5.5 over $2 y$ & 3.0 & 2nd eye \\
\hline Smith et al.., ${ }^{[20]}$ UK, £, 2001 & $\begin{array}{l}\text { Govt and tx costs only; } 3 y \text { of } t x ; \\
2-\text { and } 5 \text {-y timeframes (PL) }\end{array}$ & NR & $\begin{array}{l}6.0 \text { (costs); } \\
2.0 \text { (benefits) }\end{array}$ & 2nd eye \\
\hline \multicolumn{5}{|l|}{ Cost utility of PG vs UC, no tx or PL } \\
\hline Brown et al.,, ${ }^{[11]}$ US, \$US, 2005 & $\begin{array}{l}\text { Third-party payer - health insurance; } \\
12 y \text { of tx; } 12-y \text { timeframe (no tx) }\end{array}$ & 18.3 over $12 y$ & 3.0 & 2nd eye \\
\hline $\begin{array}{l}\text { Colquitt et al. (HTA), }{ }^{[2]} \text { UK, } \\
£, 2005\end{array}$ & $\begin{array}{l}\text { Healthcare system and Personal } \\
\text { Social Services; } 2 \text { y of tx; } 2 \text { - and 10-y } \\
\text { timeframes (UC) }\end{array}$ & $\begin{array}{l}9 \text { in y } 1 \\
8 \text { in y } 2\end{array}$ & 3.5 & NR \\
\hline $\begin{array}{l}\text { Earnshaw et al., }{ }^{[21]} \text { Canada, \$Can, } \\
2004\end{array}$ & $\begin{array}{l}\text { Healthcare system; } 2 \mathrm{y} \text { of tx; lifetime } \\
\text { timeframe (UC) }\end{array}$ & $\begin{array}{l}8.4 \text { in y } 1 \\
6.9 \text { in y } 2\end{array}$ & 3.0 & 2nd eye \\
\hline Javitt et al.,"[22] US, \$US, 2006 & $\begin{array}{l}\text { Third-party payer; } 2 \text { y of tx; lifetime } \\
\text { timeframe (PG/PDT vs PDT/UC) }\end{array}$ & $\begin{array}{l}8.4 \text { in y } 1 \\
6.9 \text { in y } 2\end{array}$ & 3.0 & 2nd eye \\
\hline Wolowacz et al., ${ }^{[23]}$ UK, $£, ~ N R$ & $\begin{array}{l}\text { UK Govt; } 2 \mathrm{y} \text { of tx; } 10 \text {-y timeframe } \\
\text { (BSC) }\end{array}$ & 12.6 over $2 y$ & 3.5 & 2nd eye \\
\hline \multicolumn{5}{|l|}{ Cost utility of RB vs UC, no tx or PL } \\
\hline Brown et al.., ${ }^{[24]}$ US, \$US, 2006 & $\begin{array}{l}\text { Third-party payer - health insurance; } \\
2 y \text { of tx; } 12-y \text { timeframe (sham tx) }\end{array}$ & 22 over $2 y$ & 3.0 & $\begin{array}{l}\text { 1st, 2nd and } \\
\text { combined eye }\end{array}$ \\
\hline $\begin{array}{l}\text { Colquitt et al. (HTA), }{ }^{[2]} \text { UK, } \\
£, 2005\end{array}$ & $\begin{array}{l}\text { Healthcare system and Personal } \\
\text { Social Services; } 1 \text { y of tx } \\
\text { (predominantly classic CNV); } 2 \text { y of tx } \\
\text { (minimally classic CNV); } 1 \text { - to } 2 \text { - and } \\
\text { 10-y timeframes (BSC) }\end{array}$ & $\begin{array}{l}12 \text { over } 1 \text { y (predominantly } \\
\text { classic CNV); } 24 \text { over } 2 \text { y } \\
\text { (minimally classic CNV) }\end{array}$ & 3.5 & NR \\
\hline
\end{tabular}


Table II. Contd

\begin{tabular}{|c|c|c|c|c|}
\hline $\begin{array}{l}\text { Study, country, currency, } \\
\text { y of values }\end{array}$ & Perspective (comparator) & No. of tx & $\begin{array}{l}\text { Annual disc. } \\
\text { rate }(\%)\end{array}$ & Model type \\
\hline $\begin{array}{l}\text { CADTH (CDR - manufacturer's } \\
\text { model - HTA), }{ }^{[25]} \text { Canada, \$Can, } \\
2007\end{array}$ & $\begin{array}{l}\text { Third-party payer; } 1 \text { y of tx [ANCHOR } \\
\text { and PIER]; } 2 \text { y of tx [MARINA]; 10-y } \\
\text { timeframe (BSC) }\end{array}$ & $\begin{array}{l}\text { ANCHOR, PIER or } \\
\text { MARINA regimen }\end{array}$ & 5.0 & 2nd eye \\
\hline $\begin{array}{l}\text { CADTH (CDR - CADTH model }{ }^{\text {b }}- \\
\text { HTA), }{ }^{[25]} \text { Canada, } \$ \text { Can, } 2007\end{array}$ & $\begin{array}{l}\text { Third-party payer; } 1 \mathrm{y} \text { of tx [ANCHOR } \\
\text { and PIER]; } 2 \mathrm{y} \text { of tx [MARINA]; 10-y } \\
\text { timeframe (BSC) }\end{array}$ & $\begin{array}{l}\text { ANCHOR, PIER or } \\
\text { MARINA regimen }\end{array}$ & NR & 2nd eye \\
\hline Hurley et al., ${ }^{[26]}$ US, \$US, 2004 & $\begin{array}{l}\text { Societal; third party - healthcare } \\
\text { funder; } 4 \mathrm{y} \text { of } \mathrm{tx} \text {; } 2-\text { and } 10-\mathrm{y} \\
\text { timeframes (UC) }\end{array}$ & $\begin{array}{l}12 \text { in y } 1 \\
12 \text { in } y 2 \\
4 \text { in y } 3 \\
4 \text { in y } 4\end{array}$ & 3.0 & 2nd eye \\
\hline Neubauer et al.,, ${ }^{[27]}$ Germany, $€$, NR & $\begin{array}{l}\text { Societal; } 2 \mathrm{y} \text { of tx; } 10-\mathrm{y} \text { timeframe } \\
\text { (BSC) }\end{array}$ & 6 per y for $2 y$ & 5.0 & 2nd eye \\
\hline \multicolumn{5}{|c|}{ Between-treatment comparisons of cost utility } \\
\hline $\begin{array}{l}\text { Brown et al. (HTA), }{ }^{[28]} \text { Canada, } \\
\text { \$Can, NR }\end{array}$ & $\begin{array}{l}\text { Provincial healthcare provider - direct } \\
\text { costs only; } 2 \text { y of tx; life expectancy } \\
\text { timeframe (PDT vs PG; RB vs PG) }\end{array}$ & $\begin{array}{l}\text { PDT: } 2 \text { per y } \\
\text { PG: } 8 \text { per y } \\
\text { RB: } 12 \text { per y }\end{array}$ & 5.0 & 2nd eye \\
\hline $\begin{array}{l}\text { Colquitt et al. (HTA), }{ }^{[2]} \text { UK, } £, \\
2005\end{array}$ & $\begin{array}{l}\text { Healthcare system and Personal } \\
\text { Social Services; } 1 \text { y of tx; } 1 \text { - to } 2-\text { and } \\
\text { 10-y timeframes (RB vs PDT) }\end{array}$ & $\begin{array}{l}\text { RB: } 12 \text { over } 1 \text { y } \\
\text { PDT: average no. reported } \\
\text { in ANCHOR }\end{array}$ & 3.5 & NR \\
\hline $\begin{array}{l}\text { CADTH (CDR - manufacturer's } \\
\text { model - HTA), }{ }^{[25]} \text { Canada, } \$ \text { Can, } \\
2007\end{array}$ & NR; 1 y of tx (RB vs PDT) & $\begin{array}{l}\mathrm{RB} \text { and } \mathrm{PDT}: \mathrm{ANCHOR} \\
\text { regimen }\end{array}$ & 5.0 & 2nd eye \\
\hline $\begin{array}{l}\text { CADTH (CDR - CADTH model } \\
\text { - HTA), }{ }^{[25]} \text { Canada, } \$ \text { Can, } 2007\end{array}$ & $\begin{array}{l}\text { NR; } 1 \text { y of tx; timeframe NR } \\
\text { (RB vs PDT) }\end{array}$ & $\begin{array}{l}\mathrm{RB} \text { and } \mathrm{PDT}: \mathrm{ANCHOR} \\
\text { regimen }\end{array}$ & 5.0 & 2nd eye \\
\hline $\begin{array}{l}\text { Earnshaw et al., }{ }^{[21]} \text { Canada, } \\
\text { \$Can, } 2004\end{array}$ & $\begin{array}{l}\text { Healthcare system; } 2 y \text { of tx; lifetime } \\
\text { timeframe (PG vs PDT) }\end{array}$ & $\begin{array}{l}\text { PG: } 8.4 \text { in y } 1 \\
6.9 \text { in y } 2 \\
\text { PDT: } 3.4 \text { in y } 1 \\
2.2 \text { in y } 2\end{array}$ & 3.0 & 2nd eye \\
\hline Fletcher et al., ${ }^{[29]}$ US, \$US, NR & $\begin{array}{l}\text { Third-party payer; } 2 \text { y of tx; } 2-y \\
\text { timeframe (RB vs BSC; PG vs BSC; } \\
\text { PDT vs BSC) }\end{array}$ & $\begin{array}{l}\text { RB: } \\
\text { (i) } 12 \text { in y } 1 ; 12 \text { in y } 2 ;^{c} \\
\text { (ii) } 6 \text { in y } 1 ; 4 \text { in y } 2 ;^{\text {d }} \\
\text { PG: } 8.4 \text { in y } 1 ; 6.9 \text { in y } 2 \text {; } \\
\text { PDT: } 3.4 \text { in y } 1 ; 2.2 \text { in y } 2\end{array}$ & 3.0 & $\begin{array}{l}\text { 1st and 2nd } \\
\text { eye }\end{array}$ \\
\hline $\begin{array}{l}\text { Hernandez-Pastor et al., }{ }^{[30]} \\
\text { Spain, } €, 2007\end{array}$ & $\begin{array}{l}\text { Third-party payer; } 2-y \text { and lifetime tx; } \\
2-y \text { and life expectancy timeframes } \\
\text { (RB vs PDT) }\end{array}$ & $\begin{array}{l}\text { RB: } 12 \text { per y } \\
\text { PDT: } 2.8 \text { in y } 1 \\
1 \text { per y thereafter }\end{array}$ & 3.0 & 2nd eye \\
\hline $\begin{array}{l}\text { Hernandez-Pastor et al., }{ }^{[31]} \\
\text { Spain, } €, 2008\end{array}$ & $\begin{array}{l}\text { Societal; lifetime tx; life expectancy } \\
\text { timeframe (RB vs } P G)\end{array}$ & $\begin{array}{l}\text { RB: } 12 \text { per } y \\
\text { PG: } 8 \text { per } y\end{array}$ & 3.5 & 2nd eye \\
\hline
\end{tabular}

a See table I for definitions of study acronyms.

b Assuming the Product Listing Agreement is implemented. In this agreement, the manufacturer covers the cost of ranibizumab if the patient requires more than nine vials in year 1 or six vials in years 2 and 3 of tx.

c MARINA regimen.

d PIER regimen.

\$A = Australian dollars; AETMIS = Agence D'évaluation Des Technologies Et Des Modes D'intervention En Santé; BSC=best supportive care; CADTH = Canadian Agency for Drugs and Technologies in Health; CDR=Common Drug Review; CNV=choroidal neovascularization; disc. = discount; Govt= government; HTA = health technology assessment; $\mathbf{N R}=$ not reported; PDT = photodynamic therapy; $\mathbf{P G}=$ pegaptanib; $\mathbf{P L}=$ placebo; $\mathbf{R B}=$ ranibizumab; $\mathbf{t x}=$ treatment(s); $\mathbf{U C}=$ usual care. 
comparator

\section{Laser photocoagulation vs UC, no tx or PL}

Brown et al., ${ }^{[10]}$ \$US

Brown et al.,[11] \$US

Busbee et al.. ${ }^{[12]}$ \$US

Cost utility of PDT vs verteporfin with UC, no tx or PL

Larouche and Rochon (AETMIS - HTA), ${ }^{[13]}$ \$Can

$$
\text { Predominantly classic CNV }
$$

Classic and occult CNV

Bansback et al.. ${ }^{[14]} £$

Brown et al., ${ }^{[11]}$ \$US

Brown et al.,[15] \$US

Donati, ${ }^{[16]} €$

Hopley et al., ${ }^{[17]} \$ \mathrm{~A} / £$

Initial visual acuity $6 / 12(20 / 40)$

Initial visual acuity 6/60 (20/200)

Meads et al. (HTA), ${ }^{[18]} £$

Sharma et al., ${ }^{[19]}$ \$US

Initial visual acuity $6 / 12(20 / 40)$

Initial visual acuity 6/60 (20/200)

Smith et al., ${ }^{[20] ~} £$

Government perspective

Visual acuity 6/12 (20/40)
NR

2012

1715

664085

664085

9381

1504

21246

15488

15277

2640

19494

12478

12478

5658

4695

NR

6490

494112

\section{1}

9500
0.186

0.246

5629

8179

0.0740

23176

49

49

\section{4}

33880

0.773

1.668

2.550

\subsection{2}

1.503

2.329

0.491

0.491

0.106

0.395

0.197

0.0311

0.0311

151179 (2y of blindness)

$2 \mathrm{y}, 86721$

$11 \mathrm{y}, 43547$

$2 \mathrm{y}, 173984$

$11 \mathrm{y}, 87197$

75580 (2y)

8823 (5y) 
Cost

intervention

Visual acuity 6/30 (20/100)

Tx costs-only perspective

Visual acuity 6/12 (20/40)

Visual acuity $6 / 30(20 / 100)$

\section{Cost utility of PG vs UC, no tx or PL}

Brown et al., ${ }^{[11]}$ \$US

Colquitt et al. (HTA), ${ }^{[2]} £$

Earnshaw et al., ${ }^{[21]} \$$ Can

Javitt et al., ${ }^{[22]}$ \$US

Early subfoveal CNV

Moderate subfoveal CNV

Late subfoveal CNV

Wolowacz et al., ${ }^{[23]} £$

\section{Cost utility of RB vs UC, no tx or PL}

Brown et al., [24] \$US

Colquitt et al. (HTA), ${ }^{[2]} £$

Predominantly classic CNV

12427

26888

Minimally classic CNV or occult CNV with no classic lesions

23902

31096

CADTH (CDR - manufacturer's model - HTA), ${ }^{[25]}$ \$Can

Predominantly classic CNV

Minimally classic CNV

Occult with no classic CNV

6173

6475

6173

1281

20016

66638

84185

28494

$8878 \quad 4590$

QALYs gained

comparator

intervention

comparator

ICER (cost per QALY gained)

$285867(2 \mathrm{y})$

$\begin{array}{rrr}0.093 & 0.980 & 285867(2 \mathrm{y})\end{array}$

15700

1.205

1.205
2.375

1.136

2.205

$89464(2 y)$

$38088(5 \mathrm{y})$

$0.995 \quad 0.980 \quad 411533(2 \mathrm{y})$

$\begin{array}{lll}0.093 & 1.999 & 68882(5 \mathrm{y})\end{array}$

24314

0.363

66978

2558

1.43

$63603(2 y)$

16600

4.15

3.89

30986 (10y)

UC, 7669

4.17

UC, 3.96

59039

55108

71393

4.75

4.44

36282

$3.38 \quad 58280$

2.77

132381

26111

3.324

3.027

8023

52652

2nd-eye model, 1.039

1 st-eye model, 0.425

Combined-eye model, 0.710

2nd-eye model, 50691

1st-eye model, 123887

Combined-eye model, 74169

$\begin{array}{rrrc}933 & 0.81 & 0.74 & 160181(1-2 \mathrm{y}) \\ 20431 & 4.15 & 3.59 & 11412(10 \mathrm{y}) \\ 1541 & 1.54 & 1.40 & 152464(1-2 \mathrm{y}) \\ 13787 & 4.79 & 4.10 & 25098(10 \mathrm{y}) \\ & & & \\ & & & \\ 74058 & 5.37 & 5.12 & 21857 \text { (ANCHOR regimen) } \\ 72720 & 5.01 & 4.77 & 12871 \text { (PIER regimen) } \\ 60445 & 4.48 & 3.14 & 9542 \\ 51158 & 5.93 & 5.57 & 10345\end{array}$

Continued next page 


d

comparator

CADTH (CDR, CADTH model ${ }^{\mathrm{C}}$ HTA), ${ }^{[25]}$ \$Can

Predominantly classic CNV

Minimally classic CNV

Occult with no classic CNV

Hurley et al., ${ }^{[26]}$ \$US

Including caregiver costs

Excluding caregiver costs

Neubauer et al., ${ }^{[27]} €$

Predominantly classic CNV

Minimally classic CNV

Occult CNV

\section{Between-treatment comparisons of cost utility}

Brown et al. (HTA), ${ }^{[28]}$ \$Can

Predominantly classic CNV

Any CNV lesion

Colquitt et al. (HTA), ${ }^{[2]} £$

CADTH (CDR - manufacturer's model - HTA), ${ }^{[25] ~ \$ C a n ~}$

CADTH (CDR - CADTH model $\left.{ }^{c}-H T A\right),{ }^{[25]}$ \$Can

Earnshaw et al., ${ }^{[21]} \$ \mathrm{Can}$

Fletcher et al.,[29] $\$$ US

Modified version of manufacturer's model

78900
205800
56700
88800

(2)

26619 (ANCHOR regimen)

21148 (PIER regimen)

48917

52678

56700

42700

238300

0.118

0.68

0.118

$308400(2 \mathrm{y})$

Dominant (10y)

$432900(2 \mathrm{y})$

$91900(10 y)$

6697
8010

8010
8826

0.40

0.34

16882

24766

26170

\begin{tabular}{|c|c|c|c|c|}
\hline 102472 & 96975 & 5.60 & 5.98 & $P G$ is dominant \\
\hline 140706 & 96975 & 6.75 & 5.98 & 56382 \\
\hline 138733 & 97569 & 6.72 & 5.98 & 56194 \\
\hline 12427 & 4182 & 0.81 & 0.77 & $202450(1-2 y)$ \\
\hline 26888 & 21498 & 4.15 & 3.81 & 15638 (10y) \\
\hline 79512 & 78666 & 5.37 & 5.17 & 4167 \\
\hline NR & NR & NR & NR & 5191 \\
\hline \multirow[t]{2}{*}{20016} & 15345 & 4.17 & 3.87 & 49052 \\
\hline & & & & $\begin{array}{l}992103 \text { (MARINA regimen) [RB vs BSC] } \\
626938 \text { (PIER regimen) [RB vs BSC] } \\
1483973 \text { (PG vs BSC) } \\
986913 \text { (PDT vs BSC) }\end{array}$ \\
\hline 31265 & 12937 & 1.143 & 1.003 & $1312752(2 y)$ \\
\hline 163588 & 49721 & 7.412 & 4.522 & 39398 (lifetime) \\
\hline 164870 & 93664 & 6.911 & 4.474 & 29224 \\
\hline
\end{tabular}

Hernandez-Pastor et al..[31] $€$

164870

93664

6.911

4.474

29224

b See table II for details regarding the methods for each study including the year of value.

c Assuming the Product Listing Agreement is implemented. In this agreement, the manufacturer covers the cost of ranibizumab if the patient requires more than nine vials in year 1 or six vials in years 2 and 3 of $t x$.

\$A = Australian dollars; AETMIS = Agence D'évaluation Des Technologies Et Des Modes D'intervention En Santé; BSC=best supportive care; CADTH=Canadian Agency for Drugs and Technologies in Health; $\mathbf{C D R}=$ Common Drug Review; $\mathbf{C N V}=$ choroidal neovascularization; $\mathbf{H T A}=$ health technology assessment; ICER = incremental cost-effectiveness ratio; $\mathbf{N R}=$ not reported; $\mathbf{P D T}=$ photodynamic therapy; $\mathbf{P G}=$ pegaptanib; $\mathbf{P L}=$ placebo; $\mathbf{R B}=$ ranibizumab; $\mathbf{t}=$ = treatment; $\mathbf{U C}=\mathbf{u s u a l}$ care. 
Table IV. Summary of the methods and results of economic evaluations using other cost-effectiveness measures ${ }^{\mathrm{a}}$

\begin{tabular}{|c|c|c|c|c|c|}
\hline $\begin{array}{l}\text { Study, country, currency, } \\
\text { y of pricing }\end{array}$ & Perspective (comparator) & No. of $\mathrm{tx}$ & $\begin{array}{l}\text { Annual disc. } \\
\text { rate }(\%)\end{array}$ & $\begin{array}{l}\text { Model } \\
\text { type }\end{array}$ & Outcome measures and results \\
\hline \multicolumn{6}{|l|}{ PDT and verteporfin vs UC, no tx or PL } \\
\hline Donati, ${ }^{[16]}$ Switzerland, $€, N R$ & $\begin{array}{l}\text { Healthcare system; societal; } \\
\text { 3y of tx; 3-y timeframe }(P L)\end{array}$ & 3 over $3 y$ & 0.0 & NR & $\begin{array}{l}\text { PDT: cost per vision-y gained ranged from } 8239 \text { to } 10271 \\
\text { ICER ranged from } 3846 \text { to } 7416\end{array}$ \\
\hline Greiner ${ }^{\left[{ }^{[3]}\right.}$ Switzerland, SwF, 1998 & $\begin{array}{l}\text { Societal; } 6 \text { tx in } 3 y \\
\text { 3-y timeframe }(P L)\end{array}$ & $\begin{array}{l}3 \text { in } y 1 \\
2 \text { in } y 2 \\
1 \text { in y } 3\end{array}$ & NR & NR & $\begin{array}{l}\text { PDT: cost } 15 \text { 921; vision-y saved 1.068; cost per vision-y } \\
14907 \\
\text { PL: cost } 10 \text { 397; vision-y saved 0.494; cost per vision-y } \\
21047 \\
\text { ICER } 9624\end{array}$ \\
\hline $\begin{array}{l}\text { MSAC (HTA - data from sponsor })^{[35]} \\
\text { Australia, } \$ A, N R\end{array}$ & $\begin{array}{l}\text { Societal; } 2 \mathrm{y} \text { of tx; } \\
\text { timeframe NR (PL) }\end{array}$ & NR & NR & NR & $\begin{array}{l}\text { Incremental cost } 14038 \\
\text { Incremental vision-y gained } 0.0396 \\
\text { ICER } 35346\end{array}$ \\
\hline Muslera and Natal, ${ }^{[36]}$ Spain, $€$, NR & $\begin{array}{l}\text { Healthcare system; 2-y } \\
\text { and lifetime timeframes (no tx) }\end{array}$ & $\begin{array}{l}\text { Median } 5 \text { tx } \\
\text { over } 2 y\end{array}$ & 2.5 & NR & $\begin{array}{l}\text { Quality-adjusted cost per visual-acuity life-y gained } \\
\text { Visual acuity maintained for } 2 \text { y: women } 70249 \text {; } \\
\text { men } 66931 \\
\text { Visual acuity maintained for a lifetime: women (17 y) } 7794 \text {; } \\
\text { men (13 y) } 9743\end{array}$ \\
\hline Smiddy, ${ }^{[32]}$ US, \$US, 2006 & $\begin{array}{l}\text { Third-party payer; } 1 \mathrm{y} \text { of tx; } \\
\text { lifetime timeframe (no tx) }\end{array}$ & 3.4 in y 1 & NR & NR & $\begin{array}{l}\text { Predominantly or minimally classic CNV } \\
\text { Snellen lines saved 1.44; cost per Snellen line-y } 448 \\
\text { Occult CNV } \\
\text { Snellen lines saved 1.17; cost per Snellen line-y } 551\end{array}$ \\
\hline Smith et al., ${ }^{[20]}$ UK, £, 2001 & $\begin{array}{l}\text { Govt and tx costs only; } \\
3 y \text { of } t x ; 2-\text { and } \\
5 \text {-y timeframes (PL) }\end{array}$ & NR & $\begin{array}{l}6.0 \text { (costs); } \\
2.0 \text { (benefits) }\end{array}$ & 2nd eye & $\begin{array}{l}\text { Incremental cost per vision-y gained } \\
\text { Govt perspective - visual acuity } 6 / 12(20 / 40) \\
2 \text { y } 33645 ; 5 \text { y } 1685 \\
\text { Govt perspective - visual acuity } 6 / 30(20 / 100) \\
2 \text { y } 13877 ; 5 \text { y } 4402 \\
\text { Tx costs only - visual acuity } 6 / 12(20 / 40) \\
2 \text { y } 39826 ; 5 \text { y } 7275 \\
\text { Tx costs only - visual acuity } 6 / 30(20 / 100) \\
2 \text { y } 19977 ; 5 \text { y } 10180\end{array}$ \\
\hline \multicolumn{6}{|l|}{ PG vs UC, no tx or PL } \\
\hline $\begin{array}{l}\text { Earnshaw et al., }{ }^{[21]} \text { Canada, } \\
\$ C \text { Can, } 2004\end{array}$ & $\begin{array}{l}\text { Healthcare system; } 2 \mathrm{y} \text { of } \mathrm{tx} \\
\text { lifetime timeframe (UC) }\end{array}$ & $\begin{array}{l}8.4 \text { in y } 1 \\
6.9 \text { in y } 2\end{array}$ & 3 & 2nd eye & $\begin{array}{l}\text { Cost: PG } 20 \text { 016; UC 7669; PL 6737 } \\
\text { Vision-y gained: PG 3.83; UC 3.26; PL } 2.62 \\
\text { Cost per vision-y gained: } 21559\end{array}$ \\
\hline
\end{tabular}




3

type

Smiddy, ${ }^{[32]}$ US, \$US, 2006

Wolowacz et al., ${ }^{[23]}$ UK, £, NR

\section{RB vs UC, no tx or PL}

Cohen et al.., ${ }^{[37]}$ France, $€, 2006$

Colquitt et al. (HTA), ${ }^{[2]}$ UK, £, 2005
Third-party payer; $1 \mathrm{y}$ of tx; lifetime timeframe (no tx)

\section{UK Govt; $2 \mathrm{y}$ of tx; \\ 10-y timeframe (BSC)}

Societal; $1 \mathrm{y}$ of $\mathrm{tx}$

1 -y timeframe (UC)
8.3 in y 1

12.6 over $2 y$

8 over $1 \mathrm{y}$

NR

\section{Healthcare system and}

Personal Social Services;

$1 \mathrm{y}$ of tx (predominantly classic

$\mathrm{CNV}$ ); $2 \mathrm{y}$ of tx (minimally

classic CNV); 1- to 2- and

10-y timeframes (BSC)
12 over $1 \mathrm{y}$ (predominantly classic CNV); 24 over $2 y$ (minimally classic CNV)
3.5

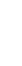

Early subfoveal CNV

PG: cost 66638 ; vision-y gained 5.26

UC: 55 108; vision-y gained 4.50

ICER 15279

Moderate subfoveal CNV

PG: cost 84 185; vision-y gained 3.75

UC: 71393 ; vision-y gained 3.12

ICER 20350

Late subfoveal CNV

PG: cost 96771 ; vision-y gained 0.42

UC: 84400 ; vision-y gained 0.21

ICER 57230

ost per Snellen line 12482

Snellen lines saved 1.04

Cost per Snellen line-y 1248

2nd eye PG: cost 28 494; vision-y gained 3.177

BSC: cost 26111 , vision-y gained 2.293

Improvement in visual acuity ( $>15$ letters on ETDRS scale) RB: cost 9123 ; success rate 0.488 ; cost per success

18721

UC: cost 7604; success rate 0.339 ; cost per success 22543

Legal blindness avoided

RB: cost 9196; success rate 0.997 ; cost per success 9224

UC: cost 5713; success rate 0.931 ; cost per success 6133

Predominantly classic CNV

RB: 1 - to 2-y costs 12427 ; vision-y saved 0.98

RB: $10-y$ costs 26888 ; vision-y saved 3.59

BSC: 1 - to 2-y costs 933; vision-y saved 0.85

BSC: $10-y$ costs 20431 ; vision-y saved 2.28

Minimally classic or occult with no classic lesions CNV

RB: 1- to 2-y costs 23 902; vision-y saved 1.87

RB: $10-y$ costs 31 096; vision-y saved 5.19

BSC: 1 - to 2-y costs 1541; vision-y saved 1.64

BSC: $10-y$ costs 13787 ; vision-y saved 3.78 


\begin{tabular}{|c|c|c|c|c|c|c|c|c|}
\hline $\begin{array}{l}\text { Study, country, currency, } \\
\text { y of pricing }\end{array}$ & Perspective (comparator) & No. of tx & $\begin{array}{l}\text { Annual disc. } \\
\text { rate }(\%)\end{array}$ & $\begin{array}{l}\text { Model } \\
\text { type }\end{array}$ & \multicolumn{4}{|c|}{ Outcome measures and results } \\
\hline Hurley et al., ${ }^{[26]}$ US, \$US, 2004 & $\begin{array}{l}\text { Societal; third party - } \\
\text { healthcare funder; } 4 \text { y of tx; } \\
\text { 2- and 10-y timeframes (UC) }\end{array}$ & $\begin{array}{l}12 \text { in y } 1 \\
12 \text { in } y 2 \\
4 \text { in y } 3 \\
4 \text { in y } 4\end{array}$ & 3.0 & 2nd eye & \multicolumn{4}{|c|}{$\begin{array}{l}\text { Incremental cost per case of blindness prevented } \\
\text { Including caregiver costs: } 2 \text { y } 145400 ; 10 \text { y dominant } \\
\text { Excluding caregiver costs: } 2 \text { y } 204100 ; 10 \text { y, } 217700 \\
\text { Incremental cost per blind-y prevented } \\
\text { Including caregiver costs: } 2 \text { y } 116500 ; 10 \text { y dominant } \\
\text { Excluding caregiver costs: } 2 \text { y } 163500 ; 10 \text { y } 29200\end{array}$} \\
\hline $\begin{array}{l}\text { CADTH (CDR - manufacturer's } \\
\text { model - HTA), }{ }^{[25]} \text { Canada, \$Can, } 2007\end{array}$ & $\begin{array}{l}\text { Third-party payer; } 1 \mathrm{y} \text { of tx } \\
\text { (ANCHOR and PIER); } \\
2 \text { y of tx (MARINA); } \\
\text { 10-y timeframe (BSC) }\end{array}$ & $\begin{array}{l}\text { ANCHOR, } \\
\text { PIER or } \\
\text { MARINA } \\
\text { regimen }\end{array}$ & 5.0 & 2nd eye & \multicolumn{4}{|c|}{$\begin{array}{l}\text { Predominantly classic CNV } \\
\text { ANCHOR regimen: vision-y gained } 2.86 \text { for RB vs } 1.81 \text { for } \\
\text { BSC; ICER } 5238 \\
\text { PIER regimen: vision-y gained } 2.68 \text { for RB vs } 1.93 \text { for } \\
\text { BSC; ICER } 4166 \\
\text { Minimally classic CNV } \\
\text { Vision-y gained } 4.48 \text { for RB vs } 3.14 \text { for BSC; ICER } 9542 \\
\text { Occult with no classic CNV } \\
\text { Vision-y gained } 5.33 \text { for RB vs } 4.00 \text { for BSC; ICER } 10345\end{array}$} \\
\hline & & & & & Regimen & $\begin{array}{l}\text { Cost } \\
\text { of } t x\end{array}$ & $\begin{array}{l}\text { Snellen } \\
\text { lines } \\
\text { saved }\end{array}$ & $\begin{array}{l}\text { Cost per Snellen line-y } \\
\text { of life expectancy }\end{array}$ \\
\hline \multirow[t]{4}{*}{ Smiddy, ${ }^{[38]}$ US, \$US, NR } & $\begin{array}{l}\text { Third-party insurer; } 2 \text { y of tx; } \\
\text { 10-y timeframe (no tx) }\end{array}$ & 22.4 over $2 y$ & NR & NR & ANCHOR & NR & $\begin{array}{l}6.2 \\
6.6\end{array}$ & $\begin{array}{l}474(1 \mathrm{y}) \\
827(2 \mathrm{y})\end{array}$ \\
\hline & & 22.4 over $2 y$ & NR & NR & MARINA & NR & $\begin{array}{l}3.5 \\
4.3\end{array}$ & $\begin{array}{l}766(1 \mathrm{y}) \\
1532(2 \mathrm{y})\end{array}$ \\
\hline & & $\begin{array}{l}6 \text { over } 1 \text { y or } \\
10 \text { over } 2 y\end{array}$ & NR & NR & PIER & $\begin{array}{l}16170 \\
26880\end{array}$ & $\begin{array}{l}3.2 \\
3.8\end{array}$ & $\begin{array}{l}505(1 \mathrm{y}) \\
707(2 \mathrm{y})\end{array}$ \\
\hline & & $\begin{array}{l}5.6 \text { over } 1 \mathrm{y} \text { or } \\
9.9 \text { over } 2 \mathrm{y}\end{array}$ & NR & NR & PrONTO & $\begin{array}{l}15472 \\
21499\end{array}$ & $\begin{array}{l}4.4 \\
4.5\end{array}$ & $\begin{array}{l}344(1 \mathrm{y}) \\
611(2 \mathrm{y})\end{array}$ \\
\hline
\end{tabular}

a See table I for study acronyms. $21499 \quad 4.5$

$611(2 \mathrm{y})$

\begin{abstract}
b PG/PDT vs PDT/UC.
\end{abstract}
$\mathbf{\$ A}=$ Australian dollars; $\mathbf{B S C}=$ best supportive care; $\mathbf{C A D T H}=$ Canadian Agency for Drugs and Technologies in Health; $\mathbf{C D R}=$ Common Drug Review; $\mathbf{C N V}=$ choroidal neovascularization; disc. $=$ discount; Govt $=$ government; HTA $=$ health technology assessment; ICER =incremental cost-effectiveness ratio; $\mathbf{M S A C}=$ Medical Services Advisory Committee; $\mathbf{N R}=$ not reported; $\mathbf{P D T}=$ photodynamic therapy; $\mathbf{P G}=$ pegaptanib; $\mathbf{P L}=$ placebo; $\mathbf{R B}=$ ranibizumab; $\mathbf{t} \mathbf{x}=$ treatment(s); $\mathbf{S w F}=\mathbf{S w i s s}$ franc; $\mathbf{U C}=$ usual care. 
initiation, but was not cost effective for patients with 'poor' initial visual acuity $(6 / 60$ [20/200] in the Australian analysis, 6/30 [20/100] in the UK analysis). In the UK analysis, PDT was shown to be cost effective for patients with 'poor' initial visual acuity when indirect costs were included. ${ }^{[20]}$

Over shorter timeframes (2-3 years), PDT was not generally shown to be cost effective compared with best supportive care, as demonstrated by studies in the UK ${ }^{[18]}$ and Switzerland. ${ }^{[16]}$ Similarly, studies that found PDT to be cost effective over $\geq 5$ years yielded ICERs considerably above accepted cost-effectiveness thresholds when time horizons of $1-2$ years were considered. ${ }^{[14,19,20]}$

\subsubsection{Other Cost-Effectiveness Measures}

Annual costs for maintaining vision over the duration of life expectancy were found to be $€ 7794$ per vision-year gained for women (17-year life expectancy) and €9743 for men (13-year life expectancy) in Spain. ${ }^{[36]}$ Data from several clinical trials, along with allowable Medicare amounts for 2006, were used to determine the cost effectiveness of PDT in the US; costs per Snellen lineyear gained were \$US448 for predominantly or minimally classic CNV and \$US551 for occult CNV. ${ }^{[32]}$

In Switzerland, two studies evaluated cost effectiveness in terms of vision-years gained and calculated costs of $€ 8239-10271$ and Swiss franc (SwF)9624 per vision-year gained over a 3-year timeframe. ${ }^{[16,34]}$

\subsection{Pegaptanib}

Pegaptanib is a pegylated, modified oligonucleotide that binds to and inhibits VEGF-A. Most studies that evaluated pegaptanib used data from the VISION study. VISION demonstrated the ability of pegaptanib to stabilize the visual acuity of patients with wet AMD. ${ }^{[7]}$ Summaries of costeffectiveness analyses of pegaptanib are presented in tables III and IV.

\subsection{Cost Utility}

A UK-based analysis by Wolowacz et al. ${ }^{[23]}$ showed treatment with pegaptanib to be cost effective relative to best supportive care for subfoveal wet $A M D$ over a 10-year timeframe from a governmental perspective; ICERs were lower when visual acuity at treatment initiation was better. However, an HTA by Colquitt et al. ${ }^{[2]}$ calculated higher costs per QALY gained over the same timeframe and from a similar perspective.

A Canadian study ${ }^{[21]}$ showed pegaptanib to be 'moderately' cost effective (\$Can20 000-100 000 per QALY gained) compared with usual care when modelled using VISION efficacy data and a lifetime timeframe.

In the US, Brown et al. ${ }^{[11]}$ showed that pegaptanib treatment of classic subfoveal CNV was 'moderately' cost effective (cost per QALY gained \$US50 000-100 000) compared with no treatment over 12 years. Javitt et al. ${ }^{[22]}$ found pegaptanib to be cost effective compared with usual care only when treatment was initiated in the early or moderate stages of disease.

\subsubsection{Other Cost-Effectiveness Measures}

Data from a number of clinical trials, along with allowable Medicare amounts for 2006, were used to determine cost effectiveness of pegaptanib in the US: cost per Snellen line-year gained was \$US1248. ${ }^{[32]}$ Incremental costs per vision-year gained varied widely, ranging from $£ 2696$ from a governmental perspective in the $\mathrm{UK}^{[23]}$ for all types of wet AMD, to \$US57230 from a third-party payer perspective (for late subfoveal $\mathrm{CNV}$ ). ${ }^{[22]}$

\subsection{Ranibizumab}

Ranibizumab is a humanized recombinant monoclonal antibody fragment directed against VEGF-A. Ranibizumab inhibits VEGF-A, thereby preventing endothelial cell proliferation and neovascularization, and slowing progression of wet AMD. In addition to stabilizing wet AMD, ranibizumab can significantly improve vision: in two pivotal randomized controlled trials (RCTs), ${ }^{[8,9]}$ more than $30 \%$ of patients with minimally classic lesions treated monthly with ranibizumab $0.5 \mathrm{mg}$, and more than $40 \%$ of patients with predominantly classic lesions treated monthly with ranibizumab $0.5 \mathrm{mg}$ plus PDT, gained $\geq 15$ letters on the ETDRS chart within 12 months; this compared with approximately $5 \%$ of those who received sham treatment or sham plus PDT, respectively. Tables III 
and IV summarize the cost-effectiveness outcomes for ranibizumab compared with usual care, no treatment or placebo.

\subsection{Cost Utility}

Ranibizumab was found to be cost effective in Canada, Germany, the UK and the US when outcomes were viewed over a long-term time horizon ( $>10$ years). Ranibizumab was also recommended by HTAs in Argentina, ${ }^{[39]}$ Australia ${ }^{[40]}$ and Scotland ${ }^{[41]}$ based on their review of the available economic evidence. Most of the studies that assessed cost effectiveness of ranibizumab used data from the MARINA $^{[9]}$ and ANCHOR ${ }^{[8]}$ studies, where intraocular injections were given on a monthly basis. However, some studies also investigated alternative regimens, such as the PIER ${ }^{[42]}$ or PrONTO regimens ${ }^{[43]}$ where ranibizumab was administered less frequently. Notably, HTAs from the $\mathrm{UK}^{[2,41]}$ reported results from the manufacturer's costeffectiveness model, which applied a dosing regimen of eight injections in the first year and six injections in subsequent years, different from those used in the MARINA and ANCHOR clinical trials. Importantly, the PrONTO study subsequently showed that less frequent dosing was effective in the majority of patients; ranibizumab was administered once monthly for 3 months and then as needed, and a mean of 5.6 injections was administered per patient over 12 months. ${ }^{[44]}$

In Germany, Neubauer et al. ${ }^{[27]}$ modelled cost effectiveness from a societal perspective using visual acuity data from the MARINA and ANCHOR clinical studies. Cost effectiveness was determined separately for the three different fluorescein angiographic subtypes of AMD included in the clinical studies, and ranibizumab was associated with a cost per QALY gained of $<€ 30000$ for each subtype. ${ }^{\text {[27] }}$

Data from the MARINA study were also used to model cost effectiveness for minimally classic/ occult CNV in a US setting, ${ }^{[24,26]}$ and ranibizumab was shown to be 'moderately' cost effective from a third-party healthcare provider perspective over a $\geq 10$-year timeframe. Moreover, in the analysis by Hurley et al., ${ }^{[26]}$ ranibizumab was found to be cost saving compared with usual care in the US when caregiver costs were taken into account.
The Canadian Agency for Drugs and Technologies in Health $(\mathrm{CADTH})^{[25]}$ conducted an independent economic evaluation of the manufacturer's cost-effectiveness data and concluded that ranibizumab treatment was cost effective compared with best supportive care if the Product Listing Agreement (where the manufacturer pays for additional treatments if patients require more than nine injections in the first year or more than six injections in the second and third years) was implemented.

The cost effectiveness (cost per QALY gained of $<£ 30000$ ) of ranibizumab was also demonstrated by an HTA in the UK for both predominantly classic CNV (data from the ANCHOR trial) and minimally classic/occult CNV (data from the MARINA trial) when costs and benefits, including cost of blindness, were considered over a 10-year timeframe. ${ }^{[2]}$ A probabilistic sensitivity analysis showed that, for patients with predominantly classic lesions, ranibizumab had a probability of being cost effective (compared with best supportive care) of $95 \%$ at a willingnessto-pay (WTP) threshold of $£ 20000$ per QALY gained and $99 \%$ at a WTP threshold of $£ 30000$ per QALY gained.

\subsubsection{Other Cost-Effectiveness Measures}

Five studies have evaluated the cost effectiveness of ranibizumab using measures other than QALYs. In France, it was concluded that ranibizumab was cost effective compared with usual care when assessed by improvements in visual acuity modelled over 1 year. The simulation used efficacy data from a number of published clinical studies and allowed patients to switch treatments if they were ineffective. Ranibizumab also reduced the rate of legal blindness, although costs per success for this endpoint were higher than with usual care. ${ }^{[37]}$ By contrast, Hurley et al. ${ }^{[26]}$ found ranibizumab to be cost saving compared with usual care over a 10-year time horizon, when evaluated by cases of blindness prevented and blind-years prevented, and viewed from a societal perspective in the US. HTAs in the UK and Canada found 2 years of monthly ranibizumab injections to be associated with incremental costs per vision-year saved of $£ 12275$ and \$Can9542, 
respectively, for minimally classic or occult with no classic $\mathrm{CNV}$, over a 10 -year timeframe; incremental costs for predominantly classic CNV were $£ 4929$ and $\$ C$ an5238, based on 1 and 2 years of ranibizumab treatment, respectively. ${ }^{[2,25]}$

Costs per Snellen line-year of life-expectancy were found to be \$US827, \$US1532 and \$US707 for the ANCHOR, MARINA and PIER regimens of ranibizumab treatment, respectively, assuming 2 years of treatment and a 10 -year timeframe. ${ }^{[38]}$ Another alternative ranibizumab regimen used in the PrONTO study, where injections were given as needed, was also evaluated and found to cost \$US611 per Snellen line-year of life-expectancy.

\section{Comparison of Treatment Options}

Studies that compared more than one treatment option for wet AMD are presented in table III. In a UK-based HTA, ranibizumab was shown to be cost effective compared with PDT for predominantly classic CNV (data from the ANCHOR trial) over a 10 -year time horizon. ${ }^{[2]}$ The CADTH also showed that ranibizumab was cost effective compared with pegaptinib, based on 1 year of treatment; cost per QALY gained with ranibizumab was approximately $\$ C a n 50000$; that is, at the commonly accepted cost-effectiveness threshold. ${ }^{[28]}$

Ranibizumab was cost effective $(<€ 30000$ per QALY gained) compared with pegaptanib for minimally classic disease from a societal perspective in a Spanish setting. ${ }^{[30,31]}$ From a third-party payer's perspective, ranibizumab treatment was slightly above the generally accepted cost-effectiveness threshold when administered according to the ANCHOR regimen; however, sensitivity analysis showed that it had a lower cost per QALY gained $(<€ 5000)$, well below the cost-effectiveness threshold, when administered as needed.

Pegaptanib was a cost-effective treatment alternative to PDT for subfoveal wet AMD in Canada when costs related to wet AMD comorbidities were taken into consideration. ${ }^{[21]}$ In a US study of patients with classic subfoveal $\mathrm{CNV},{ }^{[11]}$ laser photocoagulation was associated with a lower cost per QALY (\$US8179) than PDT (\$US31 544) and pegaptanib (\$US66 978). PDT and pegaptanib improved quality of life (QOL) to a greater extent; compared with no treatment, PDT could be considered cost effective (ICER $<$ SUS50 000 per QALY gained) and pegaptanib treatment could be considered 'moderately' cost effective (\$USUS50 000-100 000 per QALY gained).

In the US, ${ }^{[29]}$ a decision-tree analysis using a 2-year timeframe and a variety of published trial data for the cost effectiveness of individual wet AMD therapies relative to best supportive care showed that ranibizumab was associated with a lower average cost per QALY than pegaptanib and PDT when administered using the PIER regimen (table III). When using the MARINA regimen, ranibizumab had a lower ICER than pegaptanib and a similar ICER to PDT; however, because of the 2-year time horizon employed, none of the treatments analysed met the generally accepted threshold for cost effectiveness (\$US50 000-100 000 per QALY gained). An exploratory analysis over 5 years showed the importance of time horizon, in that the cost per QALY gained for best supportive care rose markedly as the proportion of blindness and related costs increased, whereas the cost per QALY gained (and hence ICER compared with best supportive care) for ranibizumab, pegaptanib and PDT fell compared with the 2-year assessment.

\section{Bevacizumab}

Bevacizumab is an anti-VEGF-A antibody licensed for the treatment of a number of cancers, including metastatic colorectal cancer, nonsquamous non-small-cell lung cancer and metastatic breast cancer. Bevacizumab is not licensed for the treatment of wet AMD; however, because its target is the same as ranibizumab and it has a lower cost once compounded into multiple divided doses from its original oncological dose, it has been used 'off-label' by many physicians. ${ }^{[45,46]}$

\section{1 Cost Utility}

There is limited information for evaluating the cost effectiveness of bevacizumab in wet AMD. Bevacizumab is often assumed to be more cost effective than ranibizumab by virtue of its lower unit cost, but to date, health economic studies 
have not compared the two treatments directly. As a consequence of the paucity of robust bevacizumab clinical data, our review identified no studies that directly compared the cost utility of bevacizumab with any other treatment for wet AMD. HTAs ${ }^{[28,39]}$ that discussed bevacizumab concluded that there was insufficient evidence to judge its suitability for the treatment of wet AMD. Fletcher et al. ${ }^{[29]}$ evaluated the available data for bevacizumab and estimated a cost per QALY gained of \$US104 748 compared with best supportive care (the lowest ICER of all comparators assessed); however, they did not directly compare the cost effectiveness of bevacizumab with other wet AMD therapies because only shortterm efficacy data were available and these were not from an RCT. The lack of robust comparative RCT data for bevacizumab was also a major limitation for an exploratory analysis by Raftery et al. ${ }^{[47]}$ This study projected the cost effectiveness of bevacizumab relative to ranibizumab for a range of relative efficacies and determined that, at the current prices, ranibizumab would have to provide 2.5 -fold greater efficacy than bevacizumab to be considered cost effective at the generally accepted threshold of $£ 30000$ per QALY gained. However, the model used a rather low cost for bevacizumab ( $£ 26$ per injection) and by assuming that the adverse effect profiles of ranibizumab and bevacizumab were equal, did not take into account the potential for differences in systemic adverse events that could affect the relative costeffectiveness ratios of these agents.

\subsection{Other Cost-Effectiveness Measures}

Smiddy ${ }^{[38]}$ used data from several open-label, non-randomized, uncontrolled treatment studies and calculated a cost per Snellen line-year of \$US84-107, depending on whether bevacizumab was administered every 6 weeks, or given as needed.

\subsection{Important Considerations for Intraocular Bevacizumab Use}

Off-label use of bevacizumab is controversial because there is currently little evidence by which to evaluate its efficacy and long-term safety in the treatment of wet AMD or other retinal diseases.
Although the recently completed ABC trial has provided evidence that long-term intravitreal bevacizumab provides visual acuity improvements superior to standard care for wet AMD (pegaptanib or PDT) with a low rate of serious ocular adverse events, ${ }^{[48]}$ there is still relatively little evidence from robust RCTs - the gold standard for evaluating the efficacy and safety of investigational therapies. A recent systematic review ${ }^{[49]}$ of bevacizumab in the treatment of ocular neovascular diseases identified 474 studies, of which nine were RCTs that employed the minimum methodological rigour necessary to generate robust data. Furthermore, methodological flaws within these trials, including potential performance bias in six of the studies and detection bias in five of the studies, precluded any definitive conclusions regarding the safety of intraocular bevacizumab administration. This lack of safety data makes it difficult at present to assess the true cost effectiveness of bevacizumab in wet AMD, at least until the large, ongoing head-to-head studies report their findings (expected in 2011-12 ${ }^{[50]}$ ).

Differences between the bevacizumab and ranibizumab molecules, and their respective formulations, could affect the safety, efficacy and costs of treatment. ${ }^{[51]}$ Unlike ranibizumab, bevacizumab was not designed for use in the eye, and there is a lack of safety data regarding its intraocular use. Ranibizumab is also formulated to optimize delivery of the active treatment, while avoiding potential ocular and systemic complications. By contrast, bevacizumab is not available at the small doses needed for intravitreal injection, and so vials need to be split for use in AMD if any cost savings are to be made. Splitting vials in this way may lead to problems maintaining sterility and potency (as there are no preservatives in the bevacizumab preparation). There is also a risk that bevacizumab may contain particulate matter that could damage the eye, as it is not manufactured for this use; this is particularly possible if vials have been repackaged by compounding pharmacies. ${ }^{[52]}$ Finally, the pharmacokinetics and pharmacodynamics of bevacizumab are different from those of ranibizumab; bevacizumab binds more weakly to the VEGF-A protein, and is a much larger molecule than rani- 
bizumab (149 kDa compared with $48 \mathrm{kDa})$ with poorer penetration through the retinal layers. ${ }^{[53]}$ Animal studies have shown systemic drug exposure to be greater with bevacizumab than ranibizumab, and so concerns have been raised over the risk of arterio-thromboembolic events. ${ }^{\text {[54-56] }}$

Recently, the French health products safety agency (AFSSAPS) published an information sheet ${ }^{[57]}$ recommending caution in the off-label use of bevacizumab: it highlighted the lack of safety data on the use of bevacizumab in ophthalmology and notes under-notification of adverse effects with the drug. Ocular inflammation, acute vision loss, and tearing or detachment of the retinal pigment epithelium are known risks, ${ }^{[58]}$ and physicians face greater legal responsibility when prescribing outside a drug licence. ${ }^{[59,60]}$

\section{Other Treatments and Treatment Combinations}

Screening for early AMD and subsequent prophylactic treatment with zinc and antioxidants was found to be cost effective for delaying and reducing progression of early AMD in Australia. ${ }^{61]}$ If savings from the reduced need for PDT were included in the model, costs per QALY gained would be lower. The model assumed optician-based screening to identify the number of people with early AMD; outcomes were modelled using data from AREDS. A US study also found prophylactic zinc and antioxidants to be cost effective for patients diagnosed with AMD as assessed by costs per QALY gained, ${ }^{[62,63]}$ Smiddy ${ }^{[32]}$ calculated costs per Snellen line-year at \$US473 for vitamin therapy.

Smiddy ${ }^{[32,38]}$ also evaluated the cost effectiveness of various treatment combinations in the US using data from various clinical trials, and allowable Medicare amounts for 2006 or 2008. Triple therapy with PDT plus corticosteroids plus an anti-VEGF-A therapy was associated with an average cost per Snellen line-year of \$US71; combinations of PDT with intravitreal triamcinolone or bevacizumab were, on average, slightly more costly at \$US66-269 per Snellen line-year, depending on how many treatment cycles were assumed, and what estimates for efficacy were used; and combination of PDT with ranibizumab was cal- culated at an average of \$US355-6195 per Snellen line-year, depending on whether treatment was for 1 or 2 years and whether ranibizumab was administered on a fixed schedule or given as needed.

\section{Health Technology Assessments}

Recommendations of formal HTAs consistently demonstrate that ranibizumab is currently a costeffective treatment option for the prevention of vision loss in wet AMD (table V). Seven HTAs conducted since 2007 have evaluated ranibizumab, only one of which did not recommend ranibizumab treatment as being cost effective; this was an assessment in Sweden that did not include a primary economic analysis and concluded that the existing literature on cost effectiveness was insufficient. ${ }^{[64]}$ Of the remaining six, two (from Scotland and Argentina) were essentially unrestricted recommendations. ${ }^{[39,41]}$ The remainder recommended use of ranibizumab with conditions to reduce the overall budget impact; three (from Canada and the UK) recommended a cap on the number of reimbursed doses, ${ }^{[2,25,28]}$ and one (from Australia) recommended limitations on patient subset and prescribing authority. ${ }^{[40]}$ Conditions set on reimbursement allowances were based on assumptions regarding the number of treatments patients would need and the costs of each treatment. In the UK, the cap was placed at 14 ranibizumab injections, mostly because the majority of injections were assumed to be administered as surgical day cases rather than outpatient procedures, thus inflating the costs and pushing cost effectiveness over the ICER threshold. ${ }^{[2]}$ In reality, most ranibizumab injections can be carried out in an outpatient setting or in private ophthalmology rooms, thus making treatment more cost effective than the HTA determined. As noted previously, the PrONTO trial subsequently showed that ranibizumab was effective under a different treatment regimen involving fewer doses on average than the regimen assessed in the UK HTA. All of the HTAs compared ranibizumab with PDT plus verteporfin; the assessments in the UK, Canada and Argentina also concluded that ranibizumab had superior cost effectiveness to pegaptanib. ${ }^{[2,28,39]}$ In addition to these appraisals, 
Table V. Health technology assessments (HTAs) of treatments (tx) for wet age-related macular degeneration (AMD)

\begin{tabular}{|c|c|c|}
\hline $\begin{array}{l}\text { Reference, } \\
\text { country, y }\end{array}$ & HTA body & $\begin{array}{l}\text { Treatments } \\
\text { evaluated }\end{array}$ \\
\hline $\begin{array}{l}\text { Brown et al., }{ }^{[28]} \\
\text { Canada, } 2008\end{array}$ & $\begin{array}{l}\text { Canadian Agency for Drugs } \\
\text { and Technologies in Health }\end{array}$ & $\begin{array}{l}\text { Ranibizumab } \\
\text { Pegaptanib } \\
\text { PDT with } \\
\text { verteporfin } \\
\text { Bevacizumab }\end{array}$ \\
\hline
\end{tabular}

Colquitt et al., [2] National Institute for Health

UK, 2008 and Clinical Excellence

Ranibizumab

Pegaptanib

PDT with

verteporfin

$\mathrm{CDR},{ }^{[25]}$

Canada, 2008

Canadian Expert Drug Advisory Committee

$\mathrm{SBU},[64]$

Sweden, 2008

PBAC, ${ }^{[40]}$

Australia, 2007

PBAC

Augustovski

et al., [39]

Instituto de Efectividad Clínica

Argentina, 2007 y Sanitaria

\section{$\mathrm{SMC},{ }^{[41]}$}

SMC

Scotland, 2007

SMC, ${ }^{[65]}$ SMC

Scotland, 2006

PBAC, [66] PBAC

Australia, 2005
Ranibizumab

PDT with

verteporfin

Ranibizumab

PDT with

verteporfin

Ranibizumab

PDT with

verteporfin

$\mathrm{PL}$

Ranibizumab

Pegaptanib

Laser

photocoagulation

PDT with

verteporfin

Ranibizumab

PDT with

verteporfin

Pegaptanib

PDT with

verteporfin

PDT with

verteporfin

PL

\section{Original health Recommendation}

economic

analysis?

Yes

\section{Ranibizumab recommended with dose cap}

Ranibizumab is recommended over pegaptanib or PDT as it demonstrates a reversal

of the degenerative process in wet AMD. However, a reduction in list price or dosing

frequency is required to be cost effective at a WTP of \$US50 000 per QALY gained;

there is limited clinical trial evidence on the efficacy and safety of bevacizumab in the tx of AMD

\section{Ranibizumab recommended with dose cap}

Ranibizumab, within its marketing authorization, is recommended as an option for the tx of wet AMD up to the cost of 14 injections in the treated eye and with specific diagnostic criteria; pegaptanib is not recommended for the tx of wet AMD

Ranibizumab recommended with dose cap

Ranibizumab is recommended for the tx of wet AMD when drug plan coverage is limited to a maximum of 15 vials per pt used to treat the better seeing affected eye

\section{Further evidence required}

Scientific evidence is insufficient to assess the cost effectiveness of ranibizumab in wet AMD. Monthly tx with ranibizumab improves vision to a substantially higher degree than PDT in follow-up $\leq 2 y$

\section{No \\ Ranibizumab recommended for restricted use}

Ranibizumab recommended for $t x$ of subfoveal CNV due to wet AMD as diagnosed by fluorescein angiography. Initial tx to be prescribed by an ophthalmologist pending specific authority approval

\section{Ranibizumab recommended}

Ranibizumab and pegaptanib are effective for the tx of all wet forms of AMD, and can be considered as first line. Ranibizumab is superior to pegaptanib as it not only delays or decreases vision loss, but also causes a great number of pts to have a significant vision improvement; use of these agents should be limited to subfoveal or juxtafoveal lesions, where laser photocoagulation is not applicable; there is not adequate evidence on the usefulness of bevacizumab

Ranibizumab recommended

Ranibizumab is recommended for the tx of wet AMD. It should be stopped if visual acuity falls persistently below $6 / 60$ during $t x$

Pegaptanib recommended for restricted pt subset

Pegaptanib is recommended for the tx of wet AMD in pts with visual acuity between $6 / 12$ and $6 / 60$ (inclusive) and should be stopped if visual acuity falls below $6 / 60$ during tx or where severe visual loss is experienced

\section{PDT with verteporfin recommended for restricted pt subset}

PDT is recommended for subfoveal CNV secondary to wet AMD where the CNV

comprises predominantly ( $\geq 50 \%$ ) classic lesions as defined by fluorescein angiography 


\begin{tabular}{llll}
\hline $\begin{array}{l}\text { Reference, } \\
\text { country, } y\end{array}$ & HTA body & $\begin{array}{l}\text { Treatments } \\
\text { evaluated }\end{array}$ & $\begin{array}{l}\text { Original health } \\
\text { economic } \\
\text { analysis? }\end{array}$ \\
\hline AETMIS ${ }^{[13]}$ & AETMIS & $\begin{array}{l}\text { PDT with } \\
\text { verteporfin }\end{array}$ & Yes
\end{tabular}

Canada (QC),

2004

verteporfin

No tx

Meads et al.,, ${ }^{[18]}$ National Institute for Health and UK, 2003 Clinical Excellence

PDT with verteporfin PL

\section{MSAC, [35]}

MSAC

Australia, 2001

PDT with

verteporfin

$\mathrm{PL}$

ANAES, ${ }^{[68]}$

ANAES

France, 2001

Meads and Moore ${ }^{[69]}$ UK, 2003

West Midlands Health Technology Assessment Collaboration
PDT with verteporfin Laser photocoagulation Transpupillary thermotherapy Surgical tx

External radiotherapy

PDT with verteporfin $\mathrm{PL}$

\section{PDT with verteporfin recommended for restricted pt subset}

PDT is recommended as effective in slowing the progression of subfoveal wet AMD with predominantly classic CNV or pure occult CNV. The estimated budget impact for a Québec cohort is acceptable if the improvement in QOL is taken into account

\section{PDT with verteporfin recommended for restricted pt subset}

PDT is recommended for the tx of wet AMD with a confirmed diagnosis of classic with no occult subfoveal CNV and best-corrected visual acuity $6 / 60$ or better; PDT is not recommended for the tx of pts with predominantly classic subfoveal CNV associated with wet AMD

\section{PDT with verteporfin permitted for restricted pt subset}

PDT is recommended for predominantly classic subfoveal CNV and occult CNV with no classic component caused by AMD and when the neovascular process is active. Precise and careful pt selection is recommended prior to the $t x$ and when the neovascular process is active. HR-QOL impact remains to be determined

\section{Further evidence required}

There is no evidence concerning pt benefits in the long term or the cost effectiveness of tx. It is important that pts in Sweden are monitored in a uniform way that allows assessment of the ongoing tx results

PDT with verteporfin recommended for restricted pt subset

PDT is recommended only for pts with predominantly classic ( $>50 \%$ classic) subfoveal CNV secondary to wet AMD

\section{Laser photocoagulation recommended}

For the exudative forms of AMD, the tx of choice for perifoveal lesions is laser photocoagulation; for subfoveal lesions, the only applicable form of tx is PDT when visual acuity is $2 / 10$ or better

AETMIS = Agence D'évaluation Des Technologies Et Des Modes D'intervention En Santé; ANAES = National Agency For Accreditation And Evaluation In Health; CDR = Common Drug Review; $\mathbf{C N V}=$ choroidal neovasculariztion; HR-QOL = health-related QOL; MSAC=Medical Services Advisory Committee; PBAC=Pharmaceutical Benefits Advisory Committee; PDT = photodynamic therapy; $\mathbf{P L}=$ placebo; $\mathbf{p t}=$ patient(s); $\mathbf{Q O L}=$ quality of life; $\mathbf{S B U}=$ Swedish Council on Technology Assessment in Health Care; $\mathbf{S M C}=\mathrm{Scottish}$ Medicines Consortium; WTP = willingness to pay.

\section{PDT with verteporfin not recommended}

PDT with verteporfin was not cost effective for tx of wet AMD; incremental cost per QALY gained was estimated at $£ 120095$ (estimate range $£ 164579-79247$ ) when 
pegaptanib was evaluated separately by the Scottish Medicines Consortium, but was recommended for use only in a restricted patient subset (defined by baseline visual acuity). ${ }^{[65]}$ Eight older HTAs conducted before the availability of ranibizumab and pegaptanib were identified for PDT with verteporfin; of these, five (from Australia, Canada, Spain and the UK) recommended PDT only in a restricted patient subset (predominantly classic CNV, based on the available clinical evidence), ${ }^{[13,18,35,66,67]}$ and the remaining three (from France, Sweden and the UK) concluded either that PDT was not cost effective compared with other options or that further cost-effectiveness evidence was required. ${ }^{[64,68,69]}$

\section{Discussion}

In the Western world, AMD is the leading cause of severe central vision loss to the point of legal blindness or worse in people aged $\geq 50$ years. ${ }^{[70,71]}$ Wet AMD thus has a major impact on patient QOL and imposes a significant burden on healthcare systems. ${ }^{[72]} \mathrm{We}$ conducted a systematic review of studies evaluating the cost effectiveness of treatments for wet AMD in the published literature and in HTA reviews, which identified a broad range of analyses of the most commonly used therapies for wet AMD (ranibizumab, pegaptanib, bevacizumab and PDT with verteporfin), along with a smaller number of analyses of other treatments, such as laser photocoagulation and antioxidant vitamins. Although there was considerable variation in the methodology for cost-effectiveness modelling across studies, ranibizumab was consistently shown to be a cost-effective therapy for wet AMD. This finding was supported by the conclusions of independently conducted HTA reviews; of seven identified HTA appraisals that included ranibizumab, six (including HTA bodies in the UK, Canada and Australia) recommended that ranibizumab was cost effective for the treatment of wet AMD. ${ }^{[2,25,28,39-41]}$

The majority of published cost-utility analyses compared ranibizumab, pegaptanib or PDT with verteporfin with no treatment, usual care or best supportive care. For ranibizumab, four of the five studies identified (including HTA appraisals from the UK and Canada and cost-utility studies from the US and Germany) showed ranibizumab to be cost effective depending on time horizon; the cost-utility studies showed ICERs below commonly accepted thresholds (e.g. $£ 30000$ or \$US50 000 per QALY gained):[2,24,27,37] a fifth study in the US showed that ranibizumab was dominant over usual care when caregiver costs were taken into account, but not cost effective when caregiver costs were excluded. ${ }^{[26]}$ Of five pegaptanib studies, one UK analysis found pegaptanib to be clearly cost effective versus best supportive care over a 10-year time horizon; ${ }^{[23]}$ the other four studies (including a UK HTA) showed that the cost effectiveness of pegaptanib varied considerably depending on the stage of disease and time horizon. ${ }^{[2,11,21,22]}$ Nine studies provided cost-utility analyses of PDT with verteporfin compared with no treatment, usual care or best supportive care, reflecting that this is an older treatment option for wet AMD. Over time horizons of $\geq 5$ years, PDT was shown to be cost effective depending on model perspective in five studies (two in the US, two in the UK and one in Canada) $;^{[11,13-15,20]}$ two studies (one in Canada, one in Australia) ${ }^{[17,19]}$ showed that PDT was of borderline cost effectiveness in patients with good baseline visual acuity but not cost effective in patients with greater impairment at baseline; by contrast, two other studies (a UK HTA analysis and a study in Switzerland) showed that PDT was not cost effective. ${ }^{[16,18]}$

Few published studies have compared active treatments, but the results of six such studies that were identified clearly suggested ranibizumab to be a cost-effective current option for the treatment of wet AMD. Thus, HTA appraisals from the UK and Canada, and a cost-utility study in Spain suggested that ranibizumab was cost effective relative to pegaptanib or PDT with verteporfin. ${ }^{[2,28,30,31]}$ The Canadian HTA assessment and a separate cost-utility study both suggested that pegaptanib was cost effective relative to PDT with verteporfin. ${ }^{[21,28]}$ A US study by Fletcher et al., ${ }^{[29]}$ comparing the cost effectiveness of all three treatments with best supportive care, showed a lower ICER for ranibizumab relative to the other treatments, with PDT having a lower ICER 
than pegaptanib. However, because of the 2-year time horizon used, all treatments were associated with ICERs of $>\$$ US500 000 per QALY gained, well in excess of accepted thresholds for cost effectiveness. This study illustrated clearly the importance of time horizon as a key determinant of cost effectiveness, as would be expected given that most wet AMD treatment costs are incurred in the initial treatment period, whereas benefits such as avoiding blindness are gained over a considerably longer timeframe. Across the studies included in this analysis, a time horizon of $\geq 5$ years was generally necessary to demonstrate cost effectiveness at standard WTP thresholds of clinically more efficacious, but more expensive, newer treatments compared with less efficacious, older options.

In addition to cost-utility analyses, most studies included one or more of a variety of additional cost-effectiveness outcomes. The most common outcome was cost per vision-year saved, although others (such as cost per Snellen line-year of lifeexpectancy and cost per case of blindness prevented) were also evaluated. The absolute costs of such outcomes are difficult to interpret, and the variation in methodology, perspective and time horizon between studies makes cross-study comparisons perilous. Nevertheless, the general trend was for ranibizumab to be more cost effective than other treatment options. For example, in the analysis conducted by Smiddy, ${ }^{[32]}$ the calculated cost per Snellen line-year gained compared with no treatment over a lifetime in predominantly or minimally classic $\mathrm{CNV}$ was lowest for ranibizumab under the PrONTO regimen with 1 year of treatment (\$US344); this was lower than the corresponding average values observed for PDT with verteporfin (\$US448) or pegaptanib (\$US1248).

It is important to note that the PIER regimen, where ranibizumab is administered less frequently than with the MARINA and ANCHOR regimens, was consistently shown to have a lower cost per QALY gained than the monthly dosing regimens of MARINA and ANCHOR. However, mean visual acuity of patients treated using the PIER regimen is not improved from baseline after 12 months of treatment, whereas mean visual acuity is increased using the MARINA or
ANCHOR regimens. ${ }^{[8,9,42]}$ Finding a dosing regimen that maintains the high level of efficacy seen in the MARINA and ANCHOR studies while minimizing costs would benefit both patients and healthcare providers. The PrONTO study, which used an Optical Coherence Tomography-guided variable-dosing regimen, resulted in visual acuity outcomes similar to the MARINA and ANCHOR studies, with fewer intravitreal injections over a 2-year period. ${ }^{[43]}$ Further investigations will be required to determine whether this is a cost-effective option.

Almost all of the studies identified in our review utilized a second-eye treatment model for assessing the cost effectiveness or cost utility of the treatments under investigation. A second-eye model is simpler, as it assumes that the first eye has already lost vision, hence visual acuity benefits are accrued immediately after treatment. By contrast, a first-eye model presumes that a patient does not accrue treatment benefit until the second eye is affected (at which point vision is deteriorating, and treatment in the first eye becomes critically important to the patient). A second-eye model will therefore predict greater value from treatment than a first-eye model, as demonstrated by the lower ICERs in studies that compared both models. ${ }^{[24]}$ On the other hand, a first-eye model contains more complexity and uncertainty because it must incorporate modelling of progression from unilateral to bilateral disease. Most clinical studies in wet AMD have evaluated a mixture of first- and second-eye cases, reflective of clinical practice; the MICMAC observational study conducted in France, Germany and Italy showed that the use of laser photocoagulation and PDT was similar in first- and second-eye cases. ${ }^{[73]}$

The growing off-label use of bevacizumab has complicated the management of wet AMD. Intravitreal administration of bevacizumab for wet AMD remains controversial given the absence of high-quality, RCT evidence for the comparative efficacy and long-term safety of this agent relative to established treatments for wet AMD, such as ranibizumab. In addition, bevacizumab cost data are complicated by external and unregulated pricing factors, such as those related to compounding 
pharmacies. As would be expected given the lack of robust clinical and economic data for bevacizumab in this indication, our review identified no studies that have properly evaluated the cost effectiveness of bevacizumab in wet AMD. The available published analyses were based only on the lower cost of bevacizumab and assumed equivalent efficacy to ranibizumab. Until rigorous clinical evidence is provided, demonstrating the comparative efficacy and safety of intravitreal bevacizumab relative to approved treatments for wet AMD, it is not appropriate to assume that bevacizumab would be cost effective compared with ranibizumab based on the lower acquisition cost alone. Moreover, while it is hoped that ongoing trials such as CATT ${ }^{[50]}$ and IVAN ${ }^{[74]}$ will be sufficient to establish the efficacy (and in the case of IVAN, the safety profile) of bevacizumab compared with ranibizumab, it is uncertain whether these outcomes will be adequately shown.

\section{Conclusions}

Our systematic review finds that ranibizumab has consistently been shown to be cost effective for wet AMD in comparison with other currently approved wet AMD therapies (usual care including PDT with verteporfin or pegaptanib), as judged by the bulk of cost-effectiveness data from the published scientific literature and supported by the independent economic assessments of healthcare providers worldwide. Pegaptanib has been shown to be of borderline cost effectiveness, depending on the stage of disease and time horizon. Prior to the launch of VEGF inhibitors, PDT with verteporfin was recommended as being a cost-effective option for the treatment of wet AMD compared with usual/best supportive care at that time.

\section{Acknowledgements}

All authors participated in the development and writing of the manuscript, and approved the final article for publication. The authors take full responsibility for the content of the article and would like to thank Dr Annette Keith (Oxford PharmaGenesis ${ }^{\mathrm{TM}}$ Ltd) for carrying out the initial literature searches, providing the authors with an overview of the search findings, and collating and incorporating comments from all authors. This editorial assistance was funded by Novartis Pharma AG, Basel, Switzerland. This analysis was supported by Novartis Pharma AG, Basel, Switzerland.

PM has received consultancy fees from Novartis Pharma AG, Pfizer, Allergan and Solvay and has also been paid lecture fees/honoraria by these companies. LA has received an unrestricted grant from Novartis. RW is an employee of Oxford PharmaGenesis ${ }^{\mathrm{TM}} \mathrm{Ltd}$, which has received project funding from Novartis Pharma AG. MG and ST are employees of Novartis Pharma AG.

\section{References}

1. Bird AC, Bressler NM, Bressler SB, et al. An international classification and grading system for age-related maculopathy and age-related macular degeneration. The International ARM Epidemiological Study Group. Surv Ophthalmol 1995; 39: 367-74

2. Colquitt JL, Jones J, Tan SC, et al. Ranibizumab and pegaptanib for the treatment of age-related macular degeneration: a systematic review and economic evaluation. Health Technol Assess 2008 May; 12 (16): iii-iv, ix-201

3. Pizzarello LD. The dimensions of the problem of eye disease among the elderly. Ophthalmology 1987; 94: 1191-5

4. Lafuma A, Brezin A, Fagnani F, et al. Nonmedical economic consequences attributable to visual impairment: a nationwide approach in France. Eur J Health Econ 2006; 7: 158-64

5. Taylor HR, Pezzullo ML, Keeffe JE. The economic impact and cost of visual impairment in Australia. Br J Ophthalmol 2006; 90: 272-5

6. Lafuma A, Brezin A, Lopatriello S, et al. Evaluation of nonmedical costs associated with visual impairment in four European countries: France, Italy, Germany and the UK. Pharmacoeconomics 2006; 24 (2): 193-205

7. Gragoudas ES, Adamis AP, Cunningham Jr ET, et al. Pegaptanib for neovascular age-related macular degeneration. N Engl J Med 2004; 351: 2805-16

8. Brown DM, Kaiser PK, Michels M, et al. Ranibizumab versus verteporfin for neovascular age-related macular degeneration. N Engl J Med 2006; 355: 1432-44

9. Rosenfeld PJ, Brown DM, Heier JS, et al. Ranibizumab for neovascular age-related macular degeneration. $\mathrm{N}$ Engl J Med 2006; 355: 1419-31

10. Brown GC, Brown MM, Sharma S, et al. Incremental cost effectiveness of laser photocoagulation for subfoveal choroidal neovascularization. Ophthalmology 2000; 107: 1374-80

11. Brown GC, Brown MM, Brown HC, et al. A value-based medicine comparison of interventions for subfoveal neovascular macular degeneration. Ophthalmology 2007; 114 : 1170-8

12. Busbee BG, Brown MM, Brown GC, et al. CME review: a cost-utility analysis of laser photocoagulation for extrafoveal choroidal neovascularization. Retina 2003; 23: 279-87

13. Larouche K, Rochon S. Evaluation of photodynamic therapy for the treatment of exudative age-related macular degeneration (ARMD) with subfoveal neovascularization. Montreal (QC): Agence d'évaluation des technologies et des modes d'intervention en santé, 2005 [online]. Available from URL: http://www.aetmis.gouv.qc.ca/site/download. 
php?f=98a72111492a641cb55a200773a47eba [Accessed 2010 Apr 26]

14. Bansback N, Davis S, Brazier J. Using contrast sensitivity to estimate the cost-effectiveness of verteporfin in patients with predominantly classic age-related macular degeneration. Eye 2007; 21: 1455-63

15. Brown GC, Brown MM, Campanella J, et al. The costutility of photodynamic therapy in eyes with neovascular macular degeneration: a value-based reappraisal with 5-year data. Am J Ophthalmol 2005; 140: 679-87

16. Donati G. Cost-effectiveness of photodynamic therapy with verteporfin for choroidal neovascularization in agerelated macular degeneration in routine clinical practice in Switzerland. J Fr Ophtalmol 2007; 30: 837-41

17. Hopley C, Salkeld G, Mitchell P. Cost utility of photodynamic therapy for predominantly classic neovascular age-related macular degeneration. Br J Ophthalmol 2004; 88: $982-7$

18. Meads C, Salas C, Roberts T, et al. Clinical effectiveness and cost-utility of photodynamic therapy for wet age-related macular degeneration: a systematic review and economic evaluation. Health Technol Assess 2003; 7 (9): v-vi, 1-98

19. Sharma S, Brown GC, Brown MM, et al. The cost-effectiveness of photodynamic therapy for fellow eyes with subfoveal choroidal neovascularization secondary to agerelated macular degeneration. Ophthalmology 2001; 108: 2051-9

20. Smith DH, Fenn P, Drummond M. Cost effectiveness of photodynamic therapy with verteporfin for age related macular degeneration: the UK case. Br J Ophthalmol 2004; 88: $1107-12$

21. Earnshaw SR, Moride Y, Rochon S. Cost-effectiveness of pegaptanib compared to photodynamic therapy with verteporfin and to standard care in the treatment of subfoveal wet age-related macular degeneration in Canada. Clin Ther 2007; 29: 2096-106

22. Javitt JC, Zlateva GP, Earnshaw SR, et al. Cost-effectiveness model for neovascular age-related macular degeneration: comparing early and late treatment with pegaptanib sodium based on visual acuity. Value Health 2008; 11: 563-74

23. Wolowacz SE, Roskell N, Kelly S, et al. Cost effectiveness of pegaptanib for the treatment of age-related macular degeneration in the UK. Pharmacoeconomics 2007; 25 (10): 863-79

24. Brown MM, Brown GC, Brown HC, et al. A value-based medicine analysis of ranibizumab for the treatment of subfoveal neovascular macular degeneration. Ophthalmology 2008; 115: 1039-45

25. Canadian Agency for Drugs and Technologies in Health. Common drug review: ranibizumab (Lucentis ${ }^{\circledR}-$ Novartis Pharmaceuticals Canada Inc.). Indication: age-related macular degeneration (AMD). Overview of CDR clinical and pharmacoeconomic reports August 2008. Ottawa (ON): CADTH, 2008 [online]. Available from URL: http:// www.cadth.ca/media/cdr/relatedinfo/cdr_trans_Lucentis_ overview_Jul-30-08_e.pdf [Accessed 2010 May 7]

26. Hurley SF, Matthews JP, Guymer RH. Cost-effectiveness of ranibizumab for neovascular age-related macular degeneration. Cost Eff Resour Alloc 2008 Jun 24; 6: 12

27. Neubauer AS, Holz FG, Schrader W, et al. Cost-utility analysis of ranibizumab (Lucentis) in neovascular macular degeneration. Klin Monbl Augenheilkd 2007; 224: 727-32
28. Brown A, Hodge W, Kymes S, et al. Management of neovascular age-related macular degeneration: systematic drug class review and economic evaluation. Ottawa $(\mathrm{ON})$ : $\mathrm{Ca}-$ nadian Agency for Drugs and Technologies in Health, 2008

29. Fletcher EC, Lade RJ, Adewoyin T, et al. Computerized model of cost-utility analysis for treatment of age-related macular degeneration. Ophthalmology 2008; 115: 2192-8

30. Hernandez-Pastor LJ, Ortega A, Garcia-Layana A, et al. Cost-effectiveness of ranibizumab compared with photodynamic treatment of neovascular age-related macular degeneration. Clin Ther 2008; 30: 2436-51

31. Hernandez-Pastor LJ, Ortega A, Garcia-Layana A, et al. Cost-effectiveness of ranibizumab compared with pegaptanib in neovascular age-related macular degeneration. Graefes Arch Clin Exp Ophthalmol 2009; 248: 467-76

32. Smiddy WE. Relative cost of a line of vision in age-related macular degeneration. Ophthalmology 2007; 114: 847-54

33. No authors listed. Photodynamic therapy of subfoveal choroidal neovascularization in age-related macular degeneration with verteporfin: one-year results of 2 randomized clinical trials - TAP report. Treatment of Age-related macular degeneration with Photodynamic therapy (TAP) Study Group [published erratum appears in Arch Ophthalmol 2000; 118 (4): 488]. Arch Ophthalmol 1999; 117 (10): 1329-45

34. Greiner RA. Cost of care for patients with age-related macular degeneration in Switzerland and cost-effectiveness of treatment with verteporfin therapy. Semin Ophthalmol 2001; 16: 218-22

35. Australian Government, Department of Health and Ageing. Medical Services Advisory Committee (MSAC). Photodynamic therapy with verteporfin for macular degeneration. Canberra (ACT): MSAC, 2001

36. Muslera E, Natal C. Cost-effectiveness of photodynamic therapy in age-related macular degeneration. Arch Soc Esp Oftalmol 2006; 81: 199-204

37. Cohen SY, Bremond-Gignac D, Quentel G, et al. Costeffectiveness sequential modeling of ranibizumab versus usual care in age-related macular degeneration. Graefes Arch Clin Exp Ophthalmol 2008; 246: 1527-34

38. Smiddy WE. Economic implications of current age-related macular degeneration treatments. Ophthalmology 2009; 116: 481-7

39. Augustovski F, Colantonio L, Pichon Riviere A. Vascular endothelial growth factor inhibitors (pegaptanib, ranibizumab and bevacizumab) in age-related macular degeneration treatment. Buenos Aires: Institute for Clinical Effectiveness and Health Policy, 2007

40. Australian Government, Department of Health and Ageing. Pharmaceutical Benefits Advisory Committee. Public summary document: ranibizumab. Canberra (ACT): Pharmaceutical Benefits Advisory Committee, 2007 [online]. Available from URL: http://www.health.gov.au/internet/main/publish ing.nsf/Content/8273CE4F07D2021FCA2572F800047B3B/ \$File/Ranibizumab.pdf [Accessed 2010 Apr 27]

41. Scottish Medicines Consortium. Ranibizumab $10 \mathrm{mg} / \mathrm{ml}$ solution for intravitreal injection (Lucentis ${ }^{\circledR}$ ). Glasgow: Scottish Medicines Consortium, 2007

42. Regillo CD, Brown DM, Abraham P, et al. Randomized, double-masked, sham-controlled trial of ranibizumab for neovascular age-related macular degeneration: PIER study year 1. Am J Ophthalmol 2008; 145: 239-48 
43. Lalwani GA, Rosenfeld PJ, Fung AE, et al. A variabledosing regimen with intravitreal ranibizumab for neovascular age-related macular degeneration: year 2 of the PrONTO Study. Am J Ophthalmol 2009; 148: 43-58

44. Fung AE, Lalwani GA, Rosenfeld PJ, et al. An optical coherence tomography-guided, variable dosing regimen with intravitreal ranibizumab (Lucentis) for neovascular age-related macular degeneration. Am J Ophthalmol 2007; 143: 566-83

45. Steinbrook R. The price of sight: ranibizumab, bevacizumab, and the treatment of macular degeneration. N Engl J Med 2006; 355: 1409-12

46. Yang YC. Developments in treatment of AMD: therapeutic intervention - anti VEGF therapy [online]. Available from URL: http://evslarchive.moorfields.nhs.uk/amd_docs_0607/ Anti\%20VEGF\%20Tx\%20.pdf [Accessed 2010 Jun 10]

47. Raftery J, Clegg A, Jones J, et al. Ranibizumab (Lucentis) versus bevacizumab (Avastin): modelling cost effectiveness. Br J Ophthalmol 2007; 91: 1244-6

48. Tufail A, Patel PJ, Egan C, et al. Bevacizumab for neovascular age related macular degeneration (ABC trial): multicentre randomised double masked study. BMJ 2010 Jun 9; 340: c2459

49. Andriolo RB, Puga ME, Belfort Jr R, et al. Bevacizumab for ocular neovascular diseases: a systematic review. Sao Paulo Med J 2009; 127: 84-91

50. National Eye Institute (NEI). Comparison of age-related macular degeneration treatments trials: Lucentis-Avastin trial [ClinicalTrials.gov identifier NCT00593450]. US National Institutes of Health, ClinicalTrials.gov [online]. Available from URL: http://www.clinicaltrials.gov [Accessed 2010 Nov 26]

51. The Bandolier Group. Lucentis versus avastin: needs must or devil drives? [online]. Available from URL: http:// www.medicine.ox.ac.uk/bandolier/band159/b159-5.html [Accessed 2009 Oct 13]

52. Kahook MY, Liu L, Ruzycki P, et al. High-molecularweight aggregates in repackaged bevacizumab. Retina 2010; 30: 887-92

53. Olsen TW. Treatment of exudative age-related macular degeneration: many factors to consider. Am J Ophthalmol 2007; 144: 281-3

54. Bakri SJ, Snyder MR, Reid JM, et al. Pharmacokinetics of intravitreal ranibizumab (Lucentis). Ophthalmology 2007; 114: 2179-82

55. Bakri SJ, Snyder MR, Reid JM, et al. Pharmacokinetics of intravitreal bevacizumab (Avastin). Ophthalmology 2007; 114: $855-9$

56. Boyer DS, Heier JS, Brown DM, et al. A phase IIIb study to evaluate the safety of ranibizumab in subjects with neovascular age-related macular degeneration. Ophthalmology 2009; 116: 1731-9

57. Agence francaise de securite sanitaire des produits de sante (AFSSAPS). Utilisation hors AMM d'Avastin ${ }^{\circledR}$-Point d'information [online]. Available from URL: http://www. afssaps.fr/Infos-de-securite/Points-d-information-Points-detape/Utilisation-hors-AMM-d-Avastin-R-Point-d-infor mation/(language)/fre-FR [Accessed 2009 Oct 20]

58. Fung AE, Rosenfeld PJ, Reichel E. The International Intravitreal Bevacizumab Safety Survey: using the internet to assess drug safety worldwide. Br J Ophthalmol 2006; 90: 1344-9

59. Williams GA. What are the legal issues regarding the use of off-label drugs? Retina Today 2007 Jan/Feb: 43-7
60. MHRA and CHM. Hot topics: off-label use of unlicensed medicines. Prescribers' responsibilities. Drug Safety Update 2009; 2: 6-7

61. Hopley C, Salkeld G, Wang JJ, et al. Cost utility of screening and treatment for early age related macular degeneration with zinc and antioxidants. Br J Ophthalmol 2004; 88: 450-4

62. Rein DB, Saaddine JB, Wittenborn JS, et al. Technical appendix: cost-effectiveness of vitamin therapy for agerelated macular degeneration. Ophthalmology 2007; 114 : e13-20

63. Rein DB, Saaddine JB, Wittenborn JS, et al. Cost-effectiveness of vitamin therapy for age-related macular degeneration. Ophthalmology 2007; 114: 1319-26

64. Swedish Council on Technology Assessment in Health Care. Photodynamic treatment for macular degeneration. Stockholm: Swedish Council on Technology Assessment in Health Care, 2001

65. Scottish Medicines Consortium. Pegaptanib $0.3 \mathrm{mg}$, solution for intravitreal injection $\left(\right.$ Macugen $\left.^{\circledR}\right)$. Glasgow: Scottish Medicines Consortium, 2006

66. Australian Government, Department of Health and Ageing. Public summary document: verteporfin. Canberra (ACT): Pharmaceutical Benefits Advisory Committee, 2005

67. Oliva G. Photodynamic therapy in the treatment of age-related macular degeneration (update). Barcelona: Catalan Agency for Health Technology Assessment and Research, 2006

68. National Agency for Accreditation and Evaluation in Health (ANAES). Treatment of age-related macular degeneration. Paris: ANAES, 2001

69. Meads C, Moore D. The clinical effectiveness and cost utility of photodynamic therapy for age-related macular degeneration: REP Committee draft report with amendments. Birmingham: Regional Evaluation Panel (REP), 2001 [online]. Available from URL: http://www.rep.bham.ac. uk/2001/Age_related_Macular_Degeneration.pdf [Accessed 2010 Nov 26]

70. Attebo K, Mitchell P, Smith W. Visual acuity and the causes of visual loss in Australia: the Blue Mountains Eye Study. Ophthalmology 1996; 103: 357-64

71. O'Shea JG. Age-related macular degeneration: a leading cause of blindness. Med J Aust 1996; 165: 561-4

72. Owsley C, Sloane ME. Contrast sensitivity, acuity, and the perception of 'real-world' targets. Br J Ophthalmol 1987; 71: 791-6

73. Bandello F, Augustin A, Sahel JA, et al. Association between visual acuity and medical and non-medical costs in patients with wet age-related macular degeneration in France, Germany and Italy. Drugs Aging 2008; 25: 255-68

74. Royal Group of Hospitals Trust (UK). A randomised controlled trial of alternative treatments to inhibit VEGF in age-related choroidal neovascularisation [ISRCTN92166560]. ISRCTN Register [online]. Available from URL: http:// controlled-trials.com/ISRCTN92166560/ISRCTN92166560 [Accessed 2010 May 1]

Correspondence: Professor Paul Mitchell, Eye Clinic (B4A), Westmead Hospital, Hawkesbury Rd, Westmead, NSW 2145, Australia.

E-mail: paul.mitchell@sydney.edu.au 
Copyright of PharmacoEconomics is the property of ADIS International Limited and its content may not be copied or emailed to multiple sites or posted to a listserv without the copyright holder's express written permission. However, users may print, download, or email articles for individual use. 